University of Montana

ScholarWorks at University of Montana

\title{
Racial Inequality in the Distribution of Hazardous Waste: A National-Level Reassessment
}

\author{
Paul Mohai \\ Robin Saha \\ University of Montana - Missoula, robin.saha@mso.umt.edu
}

Follow this and additional works at: https://scholarworks.umt.edu/environstudies_pubs

Part of the Environmental Sciences Commons

Let us know how access to this document benefits you.

\author{
Recommended Citation \\ Mohai, Paul and Saha, Robin, "Racial Inequality in the Distribution of Hazardous Waste: A National-Level \\ Reassessment" (2007). Environmental Studies Faculty Publications. 2. \\ https://scholarworks.umt.edu/environstudies_pubs/2
}

This Article is brought to you for free and open access by the Environmental Studies at ScholarWorks at University of Montana. It has been accepted for inclusion in Environmental Studies Faculty Publications by an authorized administrator of ScholarWorks at University of Montana. For more information, please contact scholarworks@mso.umt.edu. 


\title{
Racial Inequality in the Distribution of Hazardous Waste: A National-Level Reassessment
}

\author{
PAUL MOHAI, University of Michigan \\ ROBIN SAHA, University of Montana
}

\begin{abstract}
National-level studies examining racial disparities around hazardous waste treatment, storage, and disposal facilities have been very influential in defining the academic and political debates about the existence and importance of "environmental injustice." However, these studies tend to employ methods that fail to adequately control for proximity between environmentally hazardous sites and nearby residential populations. By using GIS and applying methods increasingly used in environmental inequality research that better control for proximity, we conduct a comprehensive reassessment of racial inequality in the distribution of the nation's hazardous waste facilities. We compare the magnitude of racial disparities found with those of prior studies and test competing racial, economic, and sociopolitical explanations for why such disparities exist. We find that the magnitude of racial disparities around hazardous waste facilities is much greater than what previous national studies have reported. We also find these disparities persist even when controlling for economic and sociopolitical variables, suggesting that factors uniquely associated with race, such as racial targeting, housing discrimination, or other race-related factors are associated with the location of the nation's hazardous waste facilities. We further conclude that the more recent methods for controlling for proximity yield more consistent and definitive results than those used previously, and therefore argue for their wider utilization in environmental inequality research. Keywords: environmental justice, environmental inequality, environmental racism, racial inequality, hazardous waste, GIS.
\end{abstract}

Racial inequalities in life circumstances and outcomes have long been studied, including inequalities in education, employment, income, housing, life satisfaction, poverty, health status, and mortality (Beggs 1995; Eggebeen and Lichter 1991; Hayward et al. 2000; Hughes and Thomas 1998; James and McCammon 1997; Jargowsky 1996; McCall 2001). Since the mid1980 s, there has been increasing attention to racial inequalities in the distribution of environmental quality. Attention to this form of racial inequality began as an "environmental justice" movement emerged in the 1980s and 1990s to protest the placement of waste sites and polluting industrial facilities in predominately African American and Latino communities (Bryant and Mohai 1992; Bullard 1990; Cable and Benson 1993; Schlosberg 1999; Szasz 1995). The impacts of this movement have been significant, spurring much public and academic discourse. Indeed, interest in examining the extent of social inequalities in the distribution of

This research was supported by grants from the Sociology Program and Geography and Regional Science Program of the National Science Foundation (\#0099123) and the University of Michigan State Policy Research Fund. The authors are indebted to Professor Vicki Been of the New York University School of Law and her co-author, Francis Gupta, for providing us the names and addresses of the hazardous waste treatment, storage, and disposal facilities used in their national environmental justice studies. The authors also are grateful to Ken Guire of the Center for Statistical Consultation and Research at the University of Michigan for his helpful suggestions regarding the statistical analyses for this article, Shannon Brines of the School of Natural Resources and Environment's GIS Lab for his assistance with performing spatial regression analyses, and graduate research assistants Betsy Boatner Marsh, Sara Cohen, and Theresa Weber for their assistance compiling Census data, verifying facility locations, and conducting GIS analyses. Direct correspondence to: Paul Mohai, School of Natural Resources and Environment, University of Michigan, Ann Arbor, MI 48109-1041. E-mail: pmohai@umich.edu.

Social Problems, Vol. 54, Issue 3, pp. 343-370, ISSN 0037-7791, electronic ISSN 1533-8533.

(c) 2007 by Society for the Study of Social Problems, Inc. All rights reserved. Please direct all requests for permission to photocopy or reproduce article content through the University of California Press's Rights and Permissions website at www.ucpressjournals.com/reprintinfo/asp. DOI: 10.1525/sp.2007.54.3.343. 
environmental quality, their causes and consequences, and potential remedies has spread rapidly in the past decade, not only in sociology but across a multitude of disciplines (Brown 1997; Freudenburg 1997; Pellow 2001; Taylor 2000).

Public policy activities around this issue have also been significant, as evidenced by the U.S. Environmental Protection Agency's (EPA) attempts to formulate policies to remedy environmental injustices, including its creation of an Office of Environmental Justice, by the issuance in 1994 of Presidential Executive Order 12898 calling upon all federal agencies, not just the EPA, to take into account the environmental justice consequences of their actions, and by the introduction of numerous environmental justice bills in the U.S. Congress and many state legislatures across the country (Rechtschaffen and Gauna 2002; Ringquist 2003). At the same time, the environmental justice movement has continued to grow. Given the extraordinarily rapid rise in prominence of environmental justice as an important social issue in public and academic discourse, as well as its implications in current debates about whether race as a factor affecting life outcomes is declining in significance (see, e.g., Cancio, Evans, and Maume 1996; Hughes and Thomas 1998; Wilson 1987), the attention given by sociologists to this form of racial inequality appears warranted.

Many quantitative studies examining racial and socioeconomic disparities in the distribution of environmentally hazardous sites have been conducted over the past decade. Various approaches have been applied to assess such disparities. These approaches have tended to be of two types: (1) pollution dispersion assessments and (2) site proximity assessments. Pollution dispersion assessment studies involve collecting data about the volumes and toxicities of various air and water emissions, timing of emission releases, stack heights, wind directions and speeds, and other factors (Ash and Fetter 2004; Chakraborty and Armstrong 1997; Glickman, Golding, and Hersh 1995). From these data, estimates are made about the geographic dispersion and deposition of the toxic emissions. Census data are then employed to determine the demographic characteristics of those most likely to live where pollution and toxicity levels are concentrated. Some pollution dispersion studies have gone as far as attempting to conduct risk assessments in which human exposure and expected lifetime cancer risks are estimated (for an example, see Hamilton 1999). Obtaining complete and accurate information for modeling pollution dispersions and toxicity levels has been difficult; however, this has been especially so for risk assessments. As a result, relatively few environmental inequality studies employing pollution dispersion or risk assessment methods have been conducted.

By far, the most frequently employed approach for conducting quantitative environmental inequality analyses has been to assess the proximity of hazardous sites to nearby populations. While nearly all national-level environmental inequality studies have involved proximity assessments, all national-level studies of the distribution of hazardous waste treatment, storage, and disposal facilities (TSDFs) have done so. These studies have been very influential in spurring policy development and further research in the area of environmental justice. Although most have found these disparities to be statistically significant (Lester, Allen, and Hill 200l; Ringquist 2005; Saha and Mohai 2005), there has been considerable variation in the magnitude of racial and socioeconomic disparities found. Some studies have found no race and income disparities associated with the presence of environmentally hazardous sites and locally unwanted land uses (Anderton et al. 1994; Davidson and Anderton 2000).

In another paper, the authors (Mohai and Saha 2006) hypothesized that a likely source of these uncertainties has been wide reliance in environmental inequality research on what has been termed "unit-hazard coincidence" methodology. This approach involves selecting a pre-defined geographic unit (such as zip code areas or census tracts), determining which subset of the units is coincident with the hazard and which not, and then comparing the demographic characteristics of the two sets. Implicit in this approach are two assumptions: (1) that adverse impacts tend to be concentrated within close proximity of the hazards, and (2) that populations living within the host units are located closer to the hazard under investigation than populations living in the non-host units. However, we demonstrated that this latter 
assumption is not always the case and that, in fact, the unit-hazard coincidence method fails to control for proximity in several respects. We furthermore demonstrated how alternate methods, termed "distance-based" methods, better control for proximity and how application of these methods leads to differing results.

In this paper we extend our earlier analysis by applying distance-based methods to make a comprehensive national-level reassessment of racial inequality in the distribution of hazardous waste TSDFs. We compare our results with those of prior published national-level studies, including those by the Commission for Racial Justice (CRJ 1987), Douglas L. Anderton and associates (1994), Benjamin A. Goldman and Laura Fitton (1994), Vicki Been (1995), and John Michael Oakes, Douglas L. Anderton, and Andy B. Anderson (1996). These studies have been very influential in defining the academic and political debates about the existence and importance of racial disparities around environmentally hazardous sites. By comparing our results with these prior national-level studies, we provide further evidence of the extent that use of distance-based methods alters previous estimations about the magnitude of these disparities.

\section{Theoretical Explanations of Environmental Inequality}

A wide variety of explanations have been offered as to why environmental inequalities exist. There has been special interest in understanding whether racial disparities are largely a function of socioeconomic disparities or whether other factors associated with race are also related to the distribution of environmental hazards. This latter question, as mentioned above, is especially relevant to the wider debates about the declining significance of race (Cancio et al. 1996; Hughes and Thomas 1998; Wilson 1987). The factors hypothesized to account for the racial and socioeconomic disparities in the distribution of environmental hazards, which we briefly review, tend to fall into three categories that can be termed economic, sociopolitical, and racial (Mohai and Saha 1994; Saha and Mohai 2005).

Economic factors include industry's desire to minimize production costs by siting new facilities in places where land values and operation costs are low (Boone and Madorres 1999; Daniels and Friedman 1999; Hamilton 1995; Hird and Reese 1998; Rhodes 2003). These places may coincidentally be where low-income people and minorities live (thus resulting in disparate siting). Alternatively, the facilities, once sited, may cause a decline in property values and quality of life, motivating affluent whites to move away and the poor and people of color to move in because of increased affordability of housing (resulting in disparate post-siting demographic change). Sociopolitical factors involve imbalances in social capital and political power among communities (Bullard 1990; Hamilton 1995; Hird and Reese 1998; Pellow 2002). Disproportionate siting may occur because poor, minority communities have fewer resources to mobilize and less access to decision makers than do affluent, white communities that would enable them to effectively lobby to keep out unwanted land uses. Even without intent by government and industry to do so, white NIMBYism ("not-in-my-backyard" syndrome) may lead, therefore, by default to disproportionate placement of unwanted land uses in minority neighborhoods (Camacho 1998; Cole and Foster 2001; Saha and Mohai 2005). However, decades of systematic disinvestment in many inner city areas, combined with white flight and suburban reinvestment, have created racial and economic segregation, limited inner city development options, and environmental inequality as well (Allen 2003; Hurley 1995; Montrie 2005). Racial factors are involved if siting in minority neighborhoods is intentional. Even though it may be difficult to find a "smoking gun" of prejudicial attitudes behind siting decisions, deliberate targeting of new facilities may occur because minority communities over time have come to be recognized as the "paths of least resistance" by government and industry (Bullard and Wright 1987; Saha and Mohai 2005). Even if minority communities are not intentionally targeted for society's unwanted land uses, race may still play a role in environmental inequality because housing segregation may limit the ability of people of 
color to move away from such sites, beyond the constraints of limited incomes (Mohai and Bryant 1992; Szasz and Meuser 2000). Likewise, racial inequality in education, employment, health care, land use planning, and other societal domains can limit the social and political capital of people of color communities to prevent the siting of polluting facilities and subsequent undesirable neighborhood change (Hurley 1995; Pellow 2002; Pulido, Sidawi, and Vos 1996). The disparate environmental effects of legacies of historical racial oppression have persisted in the present era (Brook 1998; Clark 2002; Lerner 2005; Pellow 2002; Pulido 1996b). Moreover, because of the institutional and systemic nature of racial discrimination, environmental inequality is inextricably linked to other forms of racial inequality (Pulido 1996a; Saha and Mohai 2005; Stretesky and Hogan 1998).

The importance of these and other factors in accounting for inequitable environmental burdens has been examined in both quantitative and qualitative studies. Qualitative case studies examining factors associated with racial inequality have pointed to a complex interplay among class, race, land use politics, and broader societal forces that shape local economic development and decline at specific locations over time (Allen 2003; Boone and Madorres 1999; Hurley 1995; Montrie 2005; Pellow 2002; Pulido et al. 1996; Szasz and Meuser 2000). At the same time, quantitative studies have examined the broad patterns of environmental hazard locations and provided statistical tests of the relative importance of the various economic, sociopolitical, and racial factors hypothesized to account for disparities in hazard locations. Although qualitative studies have provided a detailed and nuanced understanding of causal factors, which is not possible with quantitative studies, the latter have been important in testing hypotheses about causal factors, determining the generalizability of findings, and indeed in helping to first define the problem and its extent (CRJ 1987; U.S. GAO 1983). In this way, both types of studies have informed each other and the direction of environmental justice research.

Outcomes of hypothesis tests from quantitative studies have, nevertheless, tended to be mixed. For example, some quantitative studies have found an independent effect of race on the distribution of environmental burdens (such as Hird and Reese 1998 and Mohai and Bryant 1992) while others have not (such as Anderton et al. 1994 and Hamilton 1995). These mixed results have engendered considerable attention not only among academics, but policy makers and industry seeking to determine or influence the legitimacy of the environmental justice problem (Foreman 1998). We posit that the mixed outcomes from quantitative studies are attributable to the wide use of unit-hazard coincidence methodology, which fails to adequately account for the proximity between environmentally hazardous sites and nearby residential populations, and that more definitive findings can be obtained when proximity is controlled with distance-based methods.

Below is an overview of the unit-hazard coincidence and distance-based methods and how they differ in their ability to control for proximity between environmentally hazardous sites and nearby residential populations.

\section{Unit-Hazard Coincidence versus Distance-Based Methods}

As mentioned, the unit-hazard coincidence approach has been the most commonly used in conducting environmental inequality analyses, including by the most influential national studies, such as the Commission for Racial Justice (CRJ 1987). Anderton and associates (1994), Goldman and Fitton (1994), and Been (1995). However, as demonstrated in our earlier paper (Mohai and Saha 2006), this approach fails to adequately control for proximity between environmentally hazardous sites and nearby residential populations in two principal ways. First, it does not take into account the precise geographic location of the hazardous site. It goes no further than determining whether the site is coincident with one of the geographic units of analysis. Not taken into account is the proximity of the site to its host unit's boundary 


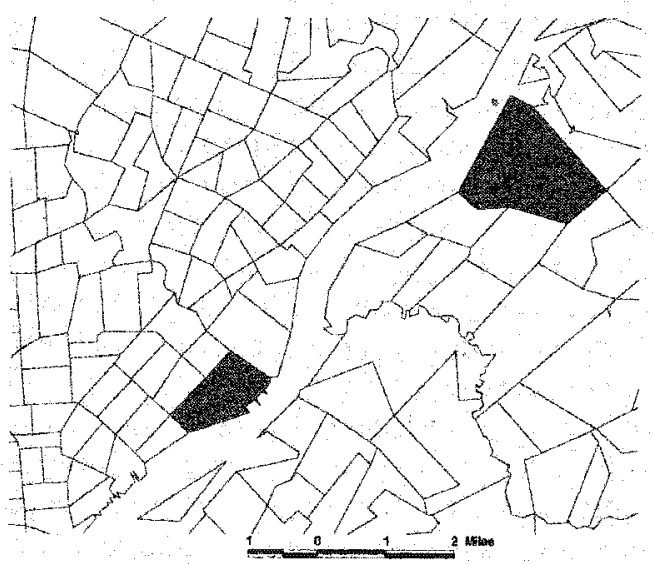

Fig. 1a-Selected TSDFs and host tracts in a large metropolitan area

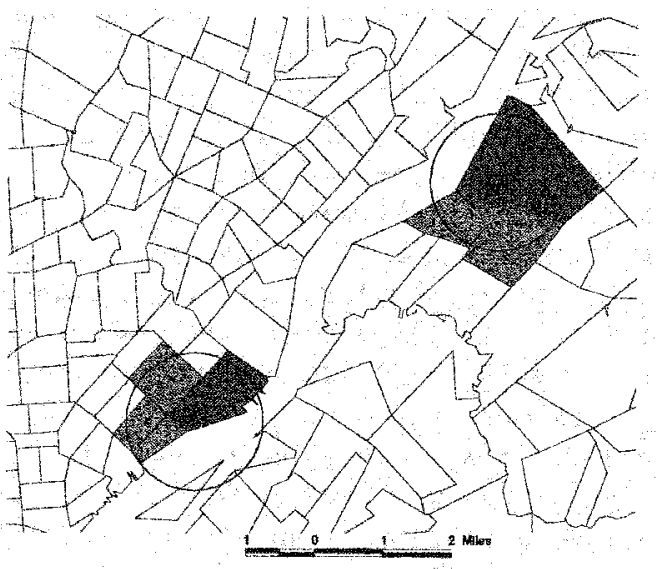

Fig. 1c - Neighborhoods within 1.0 mile of TSDFs using $50 \%$ areal containment

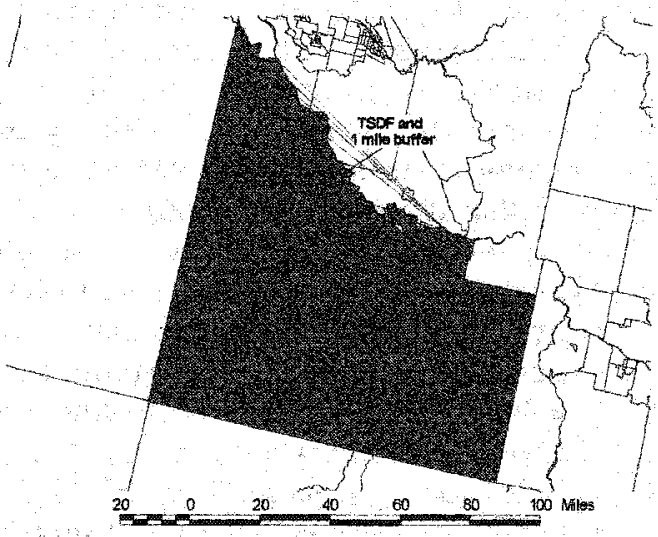

Fig. 16 - Largest host tract containing a TSDF

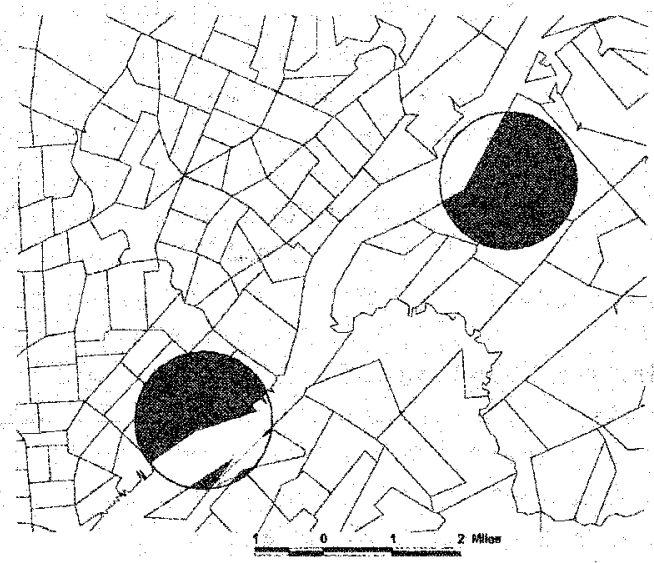

Fig. 1d - Neighborhoods within 1.0 mile of TSOFs using areal apportionment

Source: Data from U.S. Census Bureau (2000)

Figure 1 - Comparing Neighborhoods around TSDFs Identified by Unit-Hazard Coincidence and Distance-Based Methods

or its proximity to adjacent and other nearby units: However, when the precise geographic locations of hazardous sites are taken into account, it is often found that they are located near the host units' boundaries and hence very close to adjacent and other nearby units. For example, we found 49 percent, of the nation's hazardous waste TSDFs are within .25 mile of the boundary of their host census tracts, while 71 percent are within .50 mile (Mohai and Saha 2006; see also Figure la illustrating the proximity of adjacent tracts southwest of two selected TSDFs). Instead of recognizing the proximity of some of the non-host units to the hazardous sites, the unit-hazard coincidence method places nearby units in the comparison group of units, treating them no differently than non-host units much farther away. However, if there is a relationship between the presence of hazardous sites and the demographic characteristics of nearby populations, then the characteristics of nearby non-host units may be more similar to the host units proper than to non-host units farther away. 
Second, the unit-hazard coincidence method does not take into account the considerable variation in the size of the host units. It implicitly assumes that all the host units are of similar size and small enough to assure that the hazardous sites and residential populations within the units are in reasonably close proximity. However, examination of the census tracts hosting the nation's hazardous waste TSDFs reveals that this in fact is not the case. For example, we found that the smallest tract containing a hazardous waste TSDF is less than .I square mile while the largest is over 7500 square miles, with all sizes in between (Mohai and Saha 2006; see also Figure lb illustrating the largest host tract). When a host unit is small, such as the former, it can be reasonably assumed that everyone living in it is close to the site. However, when the unit is large, such as the latter, it is uncertain how many people in the unit live close by. Given that there is a greater opportunity and likelihood of people in large host units to live far from such sites, there may be less reason to expect disproportionate numbers of minorities and poor people in such units. Indeed, we found that the non-white and poverty percentages of large host tracts (those whose areas lay mostly beyond one mile of a TSDF) to be less than the non-white and poverty percentages of tracts whose areas lay mostly within one mile (Mohai and Saha 2006).

In contrast to the studies employing the unit-hazard coincidence approach, a limited number of studies have used distance-based methods in which the precise locations of the environmental hazards or locally unwanted land uses under investigation are mapped and their distances to nearby populations are controlled. The demographics of all units, not just the host unit, within a specified distance of the hazardous sites are contrasted with the demographics of units further away. Only one national level study (Hamilton and Viscusi 1999) and only two state level studies (Pollock and Vittas 1995 and Saha and Mohai 2005) of which we are aware use distance-based approaches. The remaining are focused on a single city, county, or metropolitan area (Boer et al. 1997; Chakraborty and Armstrong 1997; Glickman 1994; Mohai and Bryant 1992; Pastor, Sadd, and Hipp 2001; Sheppard et al. 1999). There have been several types of distance-based methods employed using both survey and census data.

Those involving survey data can be termed point-containment methods as the location of survey respondents and hazardous sites can be represented as points in geographic space and their distances measured. Mohai and Bryant $(1992,1998)$ provide among the earliest examples of this approach. Specifically, they mapped the location of hazardous sites and respondents to the 1990 Detroit Area Study (DAS), a metropolitan-wide probability sample survey. They then constructed circles of 1 - and 1.5-mile radii around each site and compared the demographic characteristics of respondents living within those distances to those living farther away.

Much more common in environmental inequality research than the use of survey data is the use of census data. However, census data are organized in predefined geographic units (e.g., block groups, census tracts, and zip code areas) that represent two-dimensional space rather than points. Thus, a circle with a radius of a specified distance from a hazardous site often will capture only a portion rather than all or none of the unit. One rule to decide whether or not to count a unit as within the specified distance is to include it if at least 50 percent of the unit's area is contained within the associated circle (thus the term 50 percent arealcontainment method; Mohai and Saha 2006). Alternatively, the unit can be considered within the distance if the circle contains the unit's geographic center (the centroid-containment method). Together, the captured units form the host neighborhood around the environmental hazard (see Figure Ic). The demographic characteristics of this neighborhood are derived from those of the captured units, which are either averaged or aggregated (i.e., weighted by the units' population size), and compared against the demographics of the units not captured.

An alternative to 50 percent areal containment or centroid containment methods is the areal apportionment method. Rather than including or excluding units in their entirety, depending how much area is captured, the areal apportionment method gives each unit intersected by the circle a certain weight in determining the population characteristics within the circle. Specifically, each unit's population is weighted by the proportion of the area of the unit that 
is captured by the circle. The weighted populations of these units are then aggregated to determine the demographic characteristics of perfectly circular neighborhoods around the hazard (see Figure ld).

Because the areal apportionment method weights the populations of partially intersected units by the proportion of the unit's area that is captured by a circle, it reduces the risk that any unit over (or under) influences the estimated demographic characteristics within the circle. This is an advantage over the 50 percent areal containment method where either all or none of a partially-intersected unit's population is counted depending on how much of the unit's area is captured. Nevertheless, the areal apportionment method has a limiting assumption. For the proportion of the partially captured unit's population to equal the proportion of its area that is captured, the population within the unit must be assumed to be uniformly distributed within in it, which may not be the case. However, this assumption is also made in applying other site proximity methods, including the unit-hazard coincidence method.

By applying distance-based methods, we conduct a national level reassessment of racial inequality in the distribution of hazardous waste facilities and compare these results with the results of prior national level studies that have relied on the unit-hazard coincidence approach.

\section{Data and Methods}

Studies examining racial and socioeconomic disparities in the distribution of the nation's hazardous waste TSDFs have tended to analyze somewhat different universes of facilities (Anderton et al. 1994; CRJ 1987; Been 1995; Been and Gupta 1997; CRJ 1987; Oakes et al. 1996). This is because there is no single source of information about such facilities. Thus, researchers have had to rely on a combination of various databases and sometimes not the same ones (see Been 1995 for a discussion). The fact that existing facilities close and new facilities may open in the time between studies may also partially account for the different universes of TSDFs employed in prior studies.

Since our objective is to demonstrate that any contrasting findings with prior national level studies are strictly the result of employing different methodologies (i.e., distance-based methods versus the unit-hazard coincidence method) rather than a different universe of facilities, we sought to perform our analyses on the universes of facilities used in the prior studies. Because the analyses of researchers at the Social and Demographic Research Institute (SADRI) at the University of Massachusetts (Anderton et al. 1994; Oakes et al. 1996) and the School of Law at New York University (Been 1995; Been and Gupta 1997) represent the leading (and most recent) national-level academic studies of the racial and socioeconomic inequalities around the nation's TSDFs, we requested information from both Professor Douglas Anderton (of SADRI) and Professor Vicki Been (of NYU School of Law) about the facilities used in their respective studies.

Professor Anderton provided us all the information needed to identify the host and nonhost TSDF tracts employed in the SADRI studies. However, facility names and addresses could not be released from SADRI due to confidentiality promised in surveying the companies. Professor Been was able to provide us with the names, addresses, and EPA identifiers for the 608 facilities employed in her studies. Because this information was necessary for determining the precise geographic locations of the facilities, the subsequent analyses performed in this paper are based on the universe of facilities employed in Been's studies (Been 1995; Been and Gupta 1997). These studies included all the commercial hazardous waste treatment, storage, and disposal facilities in the United States receiving off-site waste operating at the time of her studies (from the early to mid 1990s).

To identify this universe of facilities, Been relied on the U.S. Environmental Protection Agency's (EPA) Resource Conservation and Recovery Information System (RCRIS) database and 
the 1994 Environmental Services Directory (ESD).' The RCRIS database includes all hazardous waste TSDFs in the United States subject to regulation under the Resource Conservation and Recovery Act. The ESD is a commercially produced directory of hazardous waste handlers. Both databases were cross-checked by Been to determine that the facilities included in her studies were operating commercial hazardous waste TSDFs receiving waste from offsite. Where the information was ambiguous (e.g., a facility appeared in the RCRIS database as a TSDF receiving offsite waste, but was not included in ESD), phone calls were made to the companies to verify the facility's status. Phone calls were also made to the companies to verify addresses. Facility locations were matched with the 1990 census tracts in which they coincided.

Since we also needed to know the precise geographic locations of the TSDFs within the host tracts, not just that they were coincident, we performed a series of steps in order to obtain as accurate facility locations as possible. These steps included verifying facility locations and addresses and other information used to map locations (GeoLytics, Inc. 1999). We employed geographic information systems (GIS) software to geocode and map the precise locations of all 608 of Been's TSDFs. For 538 TSDFs, address and location information were obtained directly from or verified by the facility personnel. In some cases, this entailed consulting site maps obtained from the companies. For 61 TSDFs that we were not able to contact (e.g., those that were closed since Been conducted her study), state environmental agencies or the U.S. EPA were contacted for this information. For the remaining nine TSDFs for which insufficient information was available from the above sources, other sources such as former employees and online commercial mapping services were consulted. Again, because the objective of our analysis was to compare the results of different methods and studies, we used Been's entire universe of facilities and 1990 Census data, which most closely matched the time that Been's research was conducted, i.e., when each facility in her universe of TSDFs was in operation.

Once TSDF locations were established, we generated 1-, 2-, and 3-mile circular buffers around their locations. We selected these distances as they are well within the range used in prior proximity assessment studies that use distance-based methods (Anderton et al. 1994; Chakraborty and Armstrong 1997; Hamilton and Viscusi 1999; Hurley 1997; Mohai and Bryant 1992, 1998; Pastor et al. 2001; Saha and Mohai 2005). They are also within the range of distances from hazardous waste sites for which property values and health impacts have been detected (Dolk et al. 1998; Geschwind et al. 1992; Glickman et al. 1995; Kohlhase 1991; Nelson, Genereux, and Genereux 1992). In selecting this range, we furthermore wished to examine how demographic characteristics around hazardous waste TSDFs change with varying distances to these sites. We determined the demographic characteristics within the 1-, 2-, and 3 mile buffers using 1990 census data (Wessex, Inc. [1992] 1994) and applying the 50 percent areal containment and areal apportionment methods. To analyze the demographic characteristics around the nation's TSDFs, we employed 1990 digitized census areas (tracts and block groups) and zip code areas (GeoLytics, Inc. 1998; U.S. Census Bureau 1990).

Census variables examined included those used in many prior studies to assess demographic disparities and to test the relative importance of racial, economic, and sociopolitical explanations of such disparities. Race variables included percent African American, percent Hispanic, and percent persons of color. The latter variable included nonwhite racial minorities and white Hispanics and, for convenience, is hereafter referred to as percent nonwhite. Economic variables included mean household income, mean housing values, percent unemployed, and percent living below the poverty line. Variables directly measuring political activity (such as voting and participating in social movement organizations) within small geographic units (such as census tracts and block groups) are difficult to obtain. However, educational attainment and occupational status have been found to be good predictors of political resources and activity

1. Been's studies relied principally on EPA's RCRIS database to identify hazardous waste TSDFs. Prior national studies, such as those by the Commission for Racial Justice (CRJ 1987) and the Social and Demographic Research Institute at the University of Massachusetts (Anderton et al. 1994), relied principally on the ESD and earlier EPA databases. 
(Mohai 1985; Smith and Macaulay 1980) and have been used to assess sociopolitical explanations of environmental disparities in prior studies (Hamilton 1995; Hird and Reese 1998). Education and occupational status variables used in this study included percent without a high school diploma, percent with a college degree, percent employed in executive, management or professional occupations (i.e., professional "white collar" jobs), and percent employed in precision production or labor occupations (i.e., "blue collar" jobs).

\section{Results}

We found that on average the neighborhoods defined by 50 percent areal containment and areal apportionment methods, using $1-.2-$, and 3-mile radii, were much smaller than the host tracts proper (see Mohai and Saha 2006). ${ }^{2}$ For example, we found the average area of the host tracts to be 58.41 square miles, while the average areas of the host neighborhoods defined by 50 percent areal containment and areal apportionment methods were less than 3.25 square miles at the $\mathbf{1}$-mile radius and less than 28.5 square miles at the 3 -mile radius. Furthermore, the average distances of the TSDFs to their neighborhood centroids were found to be smaller for the neighborhoods defined by the distance-based methods than for the host tracts. For example, we found the average distance of TSDFs to their host tract centroids to be 1.89 miles, while for host neighborhoods defined by either of the two distance-based methods these were less than .5 mile at the 1 -mile radius and less than .75 mile at the 3 -mile radius. Thus, the neighborhoods defined by the 50 percent areal containment and areal apportionment methods are generally smaller than the host tracts proper, and the populations residing in these neighborhoods are generally closer to the TSDFs in them than are populations residing in the host tracts proper.

We therefore wanted to determine whether by using distance-based methods we would find, nationally, larger proportions of poor people and people of color living near hazardous waste TSDFs than those living farther away and whether the differences found would be greater than what has been found in prior national studies using the unit-hazard coincidence method. In making this assessment, we focus on the outcomes resulting from aggregating populations rather than averaging them across neighborhoods. This is because we were interested in knowing the demographic characteristics of host TSDF neighborhoods nationally and averaging skews the results towards the less populated neighborhoods. However, results from averaging can also be found in Table $\mathbf{l}$ and the Appendix. In order to assess whether the outcomes are affected by the size and type of geographic unit used as the building block for the host neighborhoods, we replicated the analyses three times under each distance-based method: (1) once using census tracts as the building block units, (2) again using block groups (which are generally smaller units), (3) and again using zip code areas (which are generally larger).

For purposes of providing a baseline comparison, we first examined nationally the results yielded by the unit-hazard coincidence method, i.e., we first contrasted the demographic characteristics of all host and non-host tracts in the country. As mentioned above, we were especially interested in knowing the demographic characteristics for aggregated populations and thus display these values in columns 2 and 3 of Table 1 (averaged values along with their statistical significance levels, nevertheless, are also given; columns 4 through 7). Table 1 shows that for most characteristics the differences between host and non-host tracts are not very great. For example, the difference in the nonwhite percentages between host and non-host tracts is only 1.2 percent (i.e., 25.4 percent for host tracts compared with 24.2 percent for non-host tracts).

2. For the 50 percent areal containment method, the host neighborhood is defined as the collection of tracts captured by the radius of a specified distance (i.e., 1, 2, or 3 miles). For the areal apportionment method, the host neighborhood is defined as the perfectly circular area within the radius of the specified distance. For the unit-hazard coincidence method, the neighborhood is defined as the host tract proper. See pages 12-16 and Figures 1C, 1D, and 1A, respectively. 
Table 1 - Demographic Comparisons of Aggregate and Average Populations in Host Tracts and Non-Host Tracts

\begin{tabular}{|c|c|c|c|c|c|c|}
\hline $\begin{array}{l}\text { (l) } \\
\text { Variable }\end{array}$ & $\begin{array}{c}\text { (2) } \\
\text { All Host Tracts } \\
\text { (Aggregated) }\end{array}$ & $\begin{array}{c}\text { (3) } \\
\text { All Non-Host Tracts } \\
\text { (Aggregated) }\end{array}$ & $\begin{array}{c}\text { (4) } \\
\text { Host Tracts } \\
\text { (Averaged) }\end{array}$ & $\begin{array}{c}\text { (5) } \\
\text { Non-Host Tracts } \\
\text { (Averaged) }\end{array}$ & $\begin{array}{c}\text { (6) } \\
\text { T-Test }\end{array}$ & Sign. \\
\hline Total population in $1000 \mathrm{~s}$ & 2,396 & 246,314 & 4,325 & 4,058 & 2.373 & .018 \\
\hline \multicolumn{7}{|l|}{ Race variables } \\
\hline Percent black & 12.7 & 12 & 14.4 & 13.4 & .960 & .337 \\
\hline Percent Hispanic & 10.1 & 8.8 & 10.2 & 7.8 & 3.016 & .003 \\
\hline Percent nonwhite & 25.4 & 24.2 & 27.1 & 24.4 & 2.218 & .027 \\
\hline \multicolumn{7}{|l|}{ Economic variables } \\
\hline Mean household income & $\$ 34,526$ & $\$ 38,491$ & $\$ 33,157$ & $\$ 37,310$ & -8.036 & .000 \\
\hline Mean property value & $\$ 88,892$ & $\$ 111,883$ & $\$ 82,693$ & $\$ 106,013$ & -9.467 & .000 \\
\hline Percent below poverty line & 13.6 & 13.1 & 15.6 & 14.6 & 1.872 & .061 \\
\hline Percent unemployed & 6.7 & 6.3 & 8.1 & 7.1 & 3.729 & .000 \\
\hline \multicolumn{7}{|l|}{ Sociopolitical variables } \\
\hline $\begin{array}{l}\text { Percent without a high } \\
\text { school diploma }\end{array}$ & 28.5 & 24.7 & 31.3 & 26.5 & 7.171 & .000 \\
\hline Percent with college degree & 14.4 & 20.4 & 18.7 & 25.1 & -13.405 & .000 \\
\hline $\begin{array}{l}\text { Percent employed in } \\
\text { executive, managerial, or } \\
\text { professional occupations }\end{array}$ & 21.4 & 26.4 & 19.2 & 24.6 & -13.094 & .000 \\
\hline $\begin{array}{l}\text { Percent employed in precision } \\
\text { production, trans., or labor } \\
\text { occupations }\end{array}$ & 31.4 & 26.1 & 33.5 & 27.5 & 12.918 & .000 \\
\hline
\end{tabular}

Source: Data from U.S. Census Bureau (1990)

Note: Sample sizes range from $N=546$ to 554 tracts for hosts and $N=58,670$ to 60,704 tracts for non-hosts depending on missing values.

Similarly, the difference in the percentages of those living in poverty is only .5 percent (13.6 percent for host tracts compared with 13.1 percent for non-hosts). Nevertheless, nearly all differences except for the African American percentage and percentage living in poverty are statistically significant at the 0.05 level (column 7).

Next we examined the results obtained by employing the 50 percent areal containment method (see Table 2). These results reveal that, nationally, racial and socioeconomic disparities between neighborhoods that are within and those that are beyond the 1.0 and 3.0 mile distances are much more substantial than the disparities revealed by the unit-hazard coincidence method (results for the 2 -mile radius lay in between and are thus not shown). For example, using tracts as the building block unit, the difference in the nonwhite percentages between neighborhoods within and beyond 1 mile of a TSDF is 22.2 percent (46.2 percent within 1 mile compared with 24 percent beyond 1 mile; see columns 3 and 6). At the same time, the difference in the percentages living in poverty is 7.6 percent $(20.6$ percent within 1 mile compared with 13 percent beyond). Using the generally smaller block groups reduces the disparities only slightly (a difference in the nonwhite percentages of 20.4 percent; a difference in the poverty percentages of 7 percent). Using larger zip code areas increases them slightly (a difference in the nonwhite percentages of 25.5 percent; a difference in the poverty percentages of 8.7 percent). Demographic disparities are also substantial for neighborhoods defined by a 3 -mile radius compared to areas beyond 3 miles. Differences between the host and non-host neighborhoods, defined by either 1- or 3- mile buffers, are statistically significant for all demographic characteristics, except when mean property values are estimated from zip code areas (for details of the statistical results, see Table A in Appendix). 


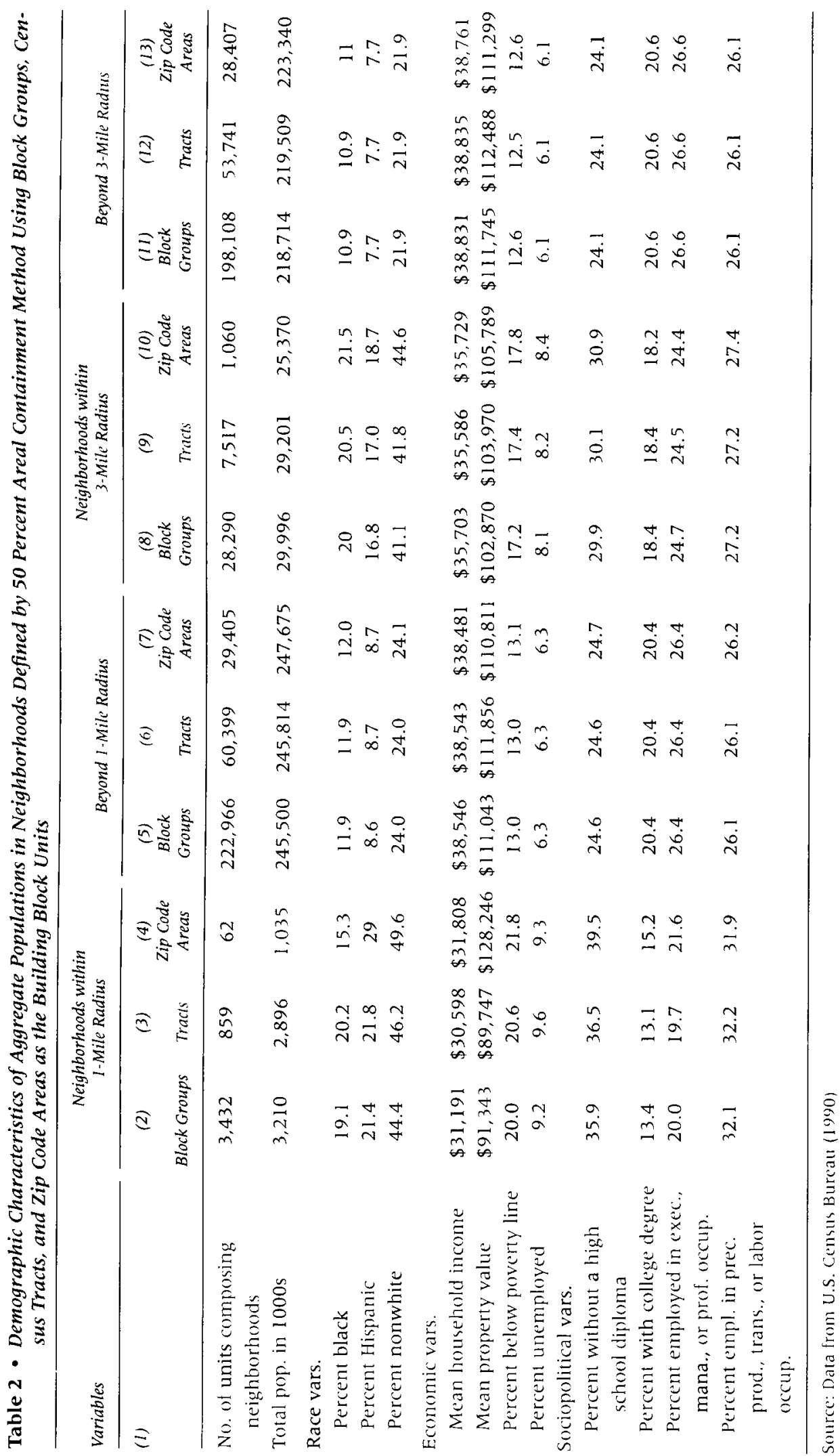


The results in Table 2 not only reveal substantially greater differences, nationally, in the demographic characteristics between host and non-host neighborhoods when the 50 percent areal containment rather than unit-hazard coincidence method is applied, they also demonstrate that the 50 percent areal containment method produces remarkable consistency in the estimates of the various demographic characteristics across the building block units (block group, census tract, and zip code area). This is especially so for results produced at the 3-mile radius. At the smaller, $\mathrm{I}$-mile radius, the results are likewise very consistent, but there are a couple of exceptions. At the 1 -mile radius, zip code areas produce a somewhat smaller African American percentage (15.3 percent) than do block groups (19.1 percent) or tracts (20.2 percent). Also, zip code areas produce a somewhat greater Hispanic percentage (29 percent compared to 21.4 percent for block groups and 21.8 percent for tracts) and greater mean housing values $(\$ 128,246$ compared to $\$ 91,343$ for block groups and $\$ 89,747$ for tracts). Because zip code areas are the largest of the three geographic units used as building blocks, we surmise that at small radii (such as 1 mile) the neighborhoods produced by them are the most likely to deviate from those of a perfect circle. The greater the deviation from a perfect circle, the less reliable the demographic results become. Nevertheless, except for the three variables mentioned, zip code areas at the I-mile radius produce results that are very similar to those obtained by using block groups and tracts as the building block units (see Figure 2 illustrating the consistency of estimates for the racial percentages using the various building block units at both the 1 - and 3 -mile radii).

We then examined the results obtained by employing the areal apportionment method (see Table 3). These results are very similar to those obtained using the 50 percent areal containment method (compare values in Tables 2 and 3; see also Figure 2), thus providing further evidence of the substantial racial and socioeconomic disparities between host and nonhost neighborhoods. However, an important improvement over the latter method is that there is greater consistency in the results across the three different building block units using areal apportionment. When this method is used, zip code areas produce results that are virtually identical to those produced by block groups and tracts for nearly all variables, even at the smaller, 1 -mile radius. For virtually all variables, estimates produced by the three units differ by no more than one or two percentage points when using areal apportionment (Table 3 and Figure 2). Differences between host and non-host areas, defined by either 1 -or 3-mile radii, are statistically significant for all demographic characteristics, including when mean property values are estimated from zip code areas (see Table A-2 in Appendix).

Although the results produced from areal apportionment are somewhat more consistent across the various building block units than those produced from 50 percent areal containment, that the results derived from the two methods are nevertheless very similar demonstrates the reliability and robustness of distance-based methods in estimating population characteristics within the small areas defined by the 1 - and 3-mile circular buffers. The above results also clearly show that, in contrast to the unit-hazard coincidence method, controlling for proximity by using distance-based methods ( 50 percent areal containment and areal apportionment) reveals substantial racial and socioeconomic disparities in the location of the nation's TSDFs. To further highlight the contrasting results obtained from using distancebased versus unit-hazard coincidence methods, we compared the above results with those obtained from the leading national studies that have analyzed the distribution of hazardous waste TSDFs by race and socioeconomic characteristics. These studies include Commission for Racial Justice (CRJ 1987), Goldman and Fitton (1994), Anderton and associates (1994), Been (1995), and Oakes, Anderton, and Anderson (1996).

Table 4 compares the values of some of the key racial and socioeconomic variables obtained from those studies. The disparities revealed are much less than those obtained by using distance-based methods. This is especially true regarding racial disparities. Indeed, Anderton and associates (1994) found that the percentage of African Americans is actually 


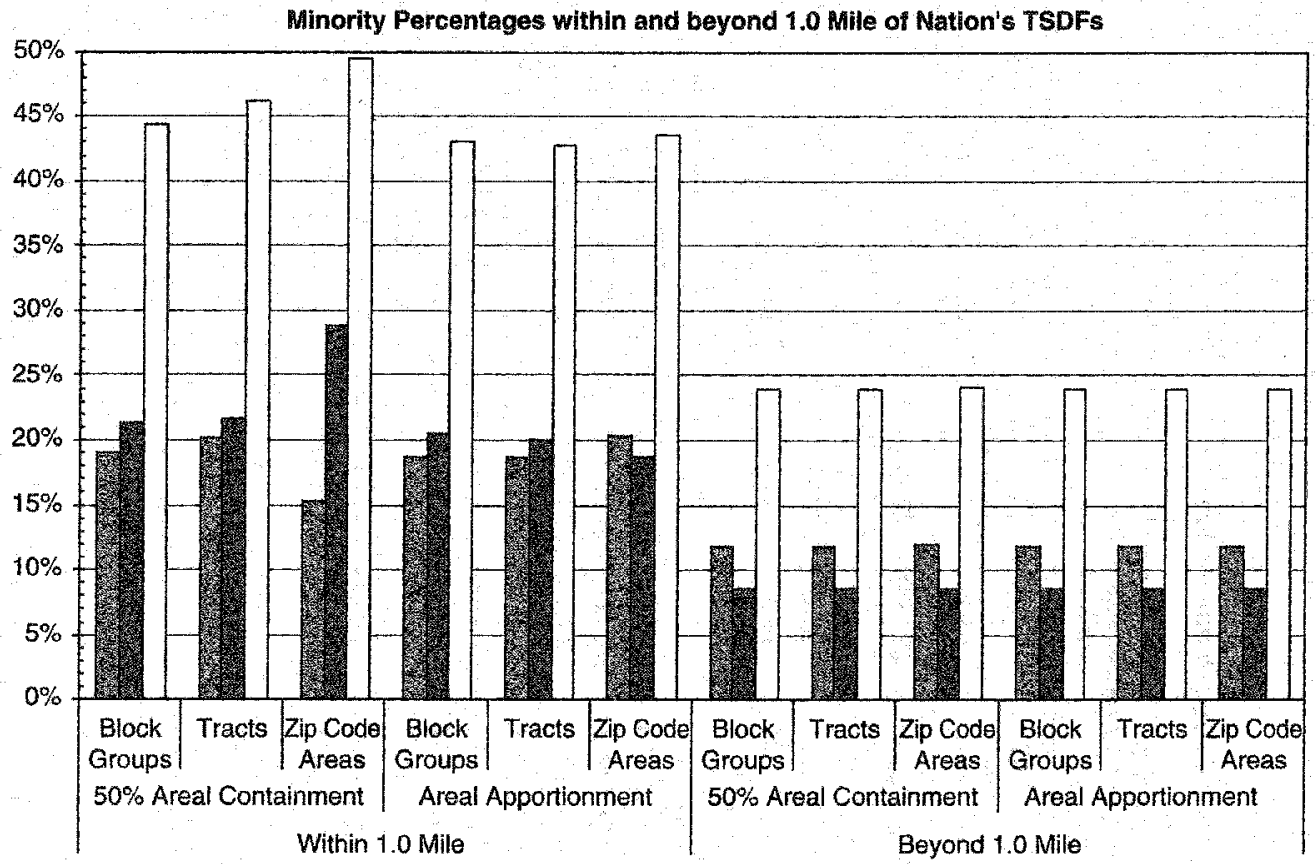

\% Black Hispanic $\quad 0 \%$ Nonwhite

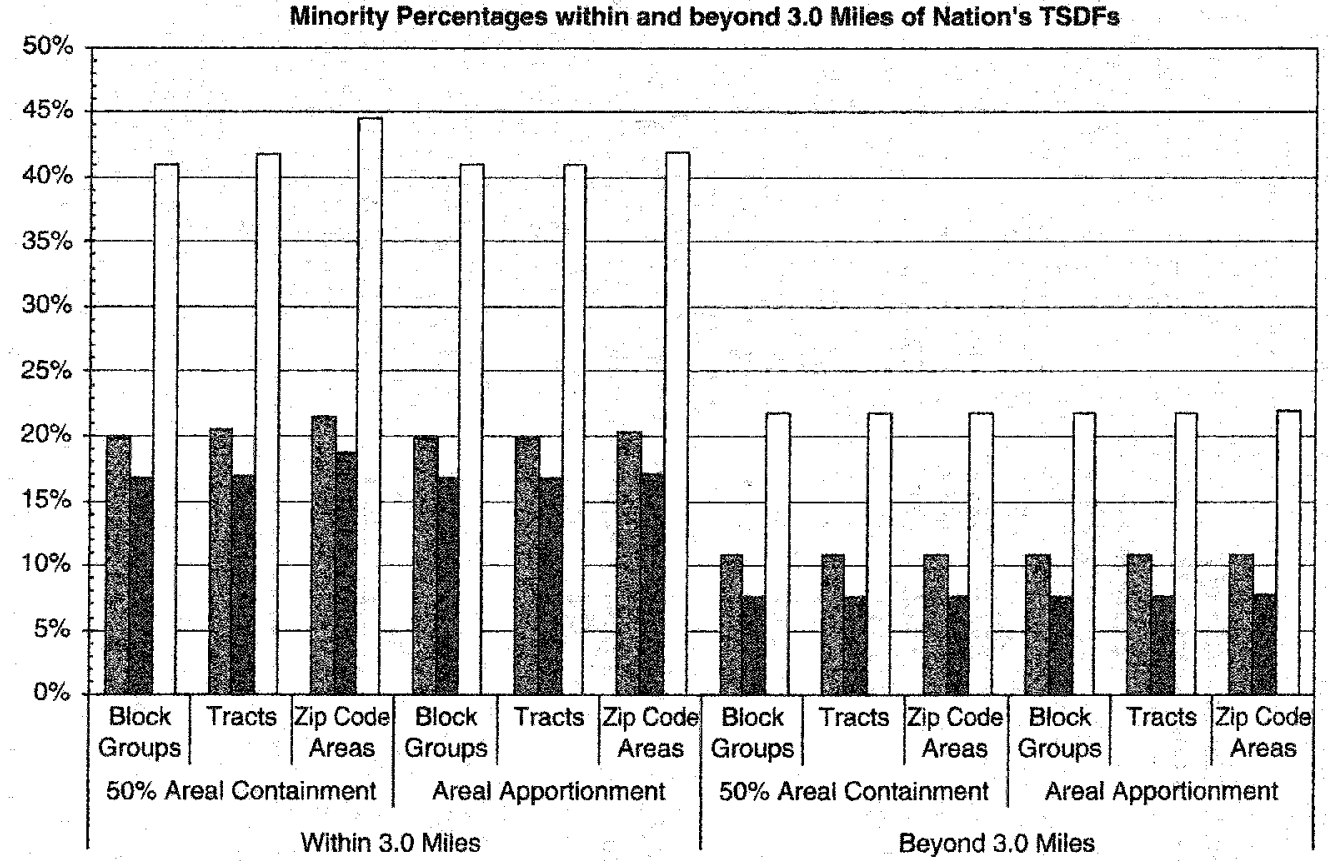

g Black $\%$ Hispanic $0 \%$ Nonwhite

Source: Data from U.S. Census Bureau (1990)

Figure 2 - Minority Percentages within and beyond Specified Distances of Nation's TSOFs Using 50 Percent Areal Containment and Areal Apportionment Methods 


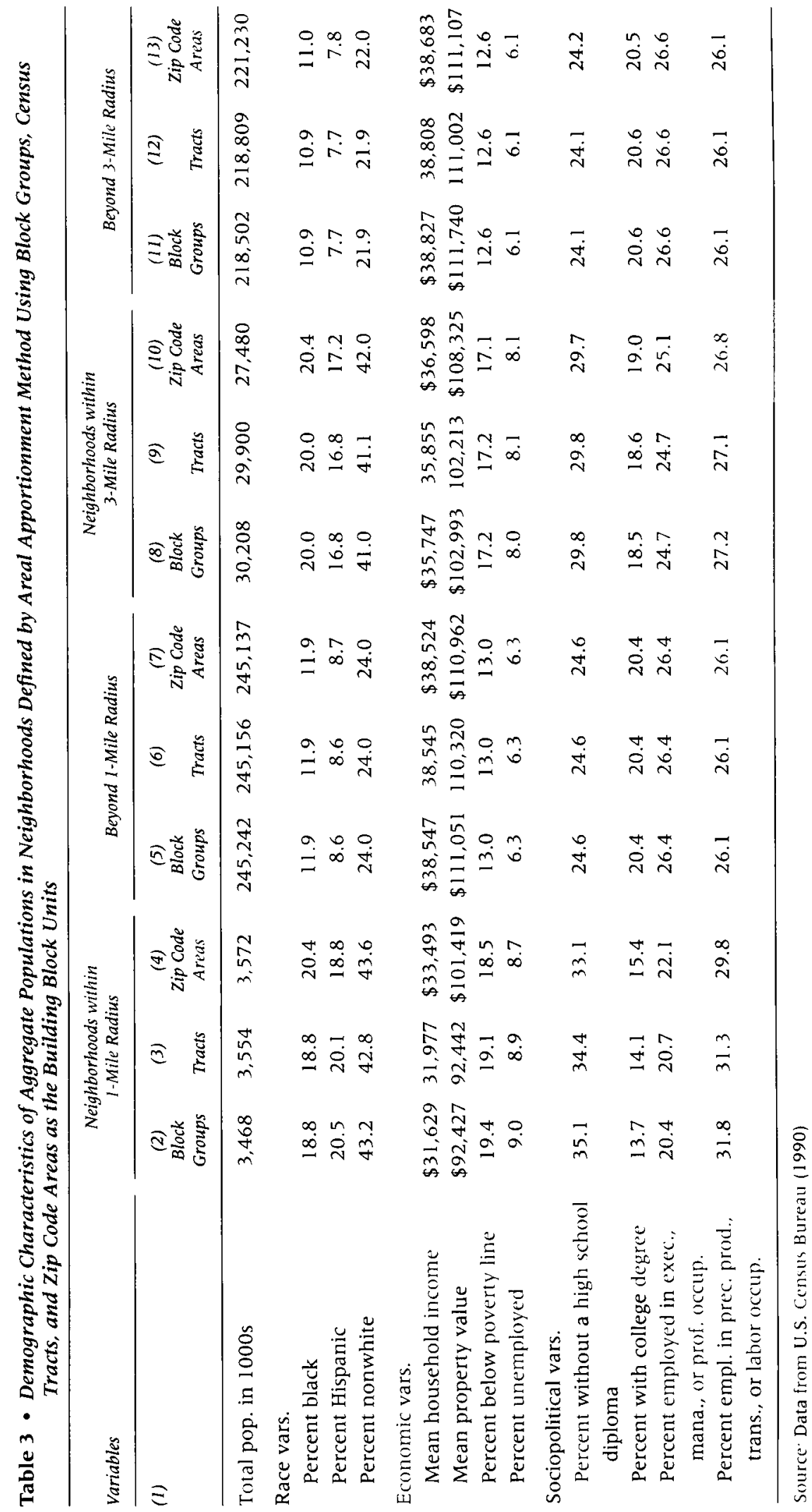




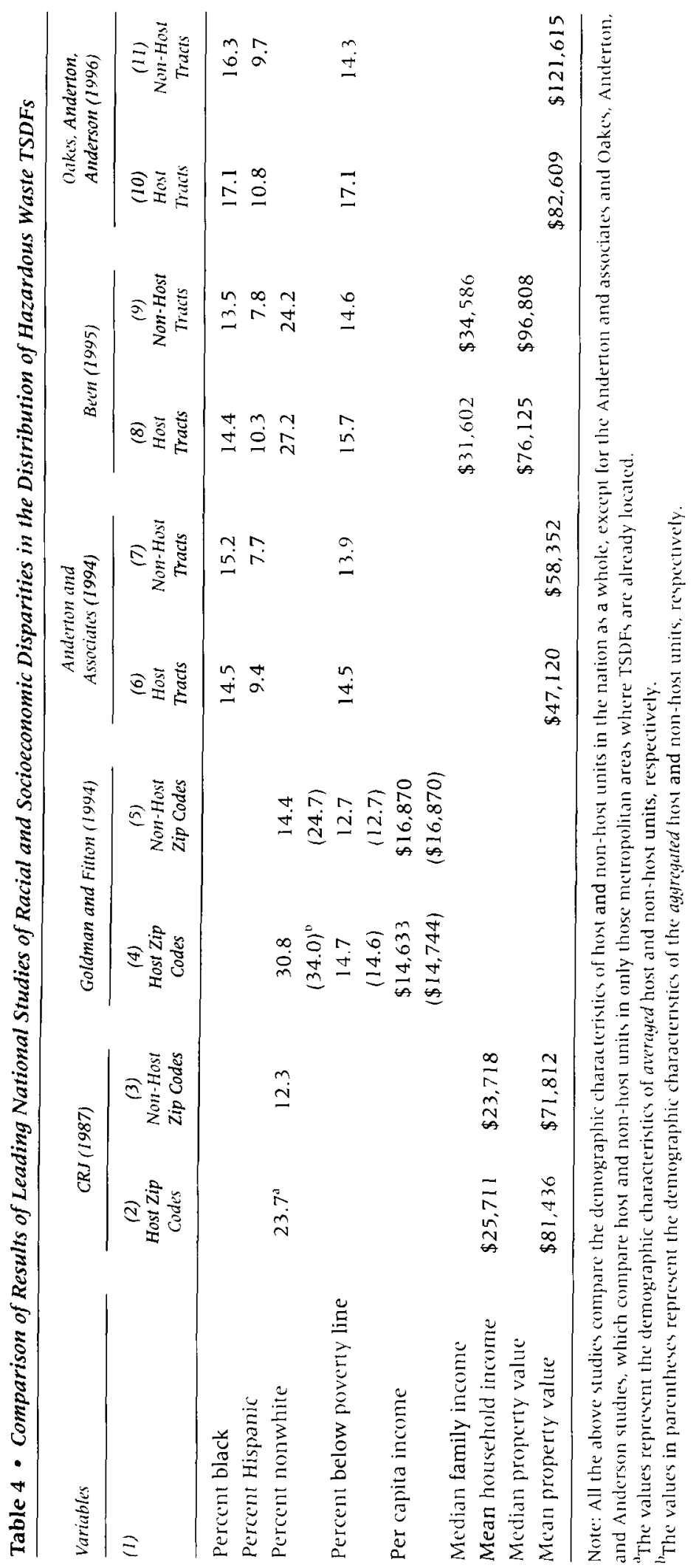


slightly less in host than in non-host tracts. ${ }^{3}$ Although the African American percentages were found to be slightly greater in host than in non-host tracts by Been (1995) and Oakes and colleagues (1996), the differences were nevertheless less than 1 percent. The CRJ (1987) and Goldman and Fitton (1994) studies found racial disparities between host and non-host zip code areas to be much greater (mean nonwhite percentages of 23.7 percent and 12.3 percent, respectively, and aggregate nonwhite percentages of 34 percent and 24.7 percent, respectively). However, these differences are still substantially smaller than those obtained by using 50 percent areal containment and areal apportionment methods.

Given that distance-based methods reveal greater racial disparities in the distribution of the nation's TSDFs than prior studies, does application of these methods also lead to different assessments about the possible underlying causes of these disparities? A comprehensive accounting of all the possible factors that may affect racial disparities in the distribution of the nation's TSDFs is beyond the scope of a single quantitative study. However, we can nevertheless assess, as many prior quantitative studies have done, the relative effect of various key economic and sociopolitical variables on the racial disparities.

To determine whether application of distance-based methods leads to different assessments about the relative importance of economic and sociopolitical factors in accounting for racial disparities in the distribution of the nation's TSDFs, logistic regression analyses were performed using unit-hazard coincidence, 50 percent areal containment, and areal apportionment methods and the results compared. ${ }^{4}$ In applying the unit-hazard coincidence method, and using census tracts as the units of analysis, the dependent variable in the logistic regression took a value of 1 if the tract hosted a TSDF and a value of 0 if it did not. In applying the 50 percent real containment method, the dependent variable took a value of 1 if the tract lay within 1 mile of a TSDF and a value of 0 if the tract lay beyond 1 mile. In applying the areal apportionment method, we used 1216 one-mile circular neighborhoods as the units of analysis. Half of these neighborhoods were centered at the 608 TSDFs. The other half were centered at 608 randomly located points within the conterminous United States. ${ }^{5}$ In the analyses, the dependent variable took a value of 1 if the neighborhood was centered at a TSDF and a value of 0 if it was centered at one of the randomly placed points. The independent variables used in all three analyses included the race, economic, and sociopolitical variables described in the methods section, excluding some of the variables (e.g., percent nonwhite, mean property values, and percent without high school diplomas) to reduce multicollinearity problems (see Table 5). Because the probability of a neighborhood hosting a locally unwanted

3. Anderton and associates (1994) and Oakes and colleagues (1996) confined their comparison of host and nonhost tracts to only those metropolitan areas already containing a TSDF. This differs from the approach of the other studies in Table 4, which compared all the host and non-host units in the nation as a whole. Furthermore, the Anderton and associates (1994) and CRJ (1987) studies employed 1980 census data, while all the other studies employed 1990 census data. These differences in approaches and data may partly account for differences in the findings.

4. In addition to the logistic regression analyses, spatial regression analyses were also performed using unit-hazard coincidence, 50 percent areal containment, and areal apportionment methods to take into account the effects of possible spatial autocorrelation. The pattern of results using spatial regression was similar to that using logistic regression, an outcome that is consistent with other studies (see, for example, Pastor, Sadd, and Morello-Frosch 2004; Mohai and Saha 2006). Spatial regression methods assume a linear model with a continuous dependent variable. However, the dependent variable in our analyses, and indeed most environmental inequality analyses, is dichotomous. Because results are not appreciably different whether using spatial or logistic regression and because the latter is the correct specification for models with a dichotomous dependent variable, we display and discuss the results of logistic regression in this article. Spatial regression results can nevertheless be obtained from the authors upon request.

5. We found that 519 TSDFs were located in metropolitan areas and 89 were located in non-metropolitan areas. In order to provide a representative sample of randomly selected points in both types of areas, we stratified the sample and generated 519 random points in the metropolitan areas and 89 in the non-metropolitan areas. We furthermore replicated our random point generation and statistical analyses two more times: (1) one additional time where the random points were distributed between metro and nonmetro areas in similar numbers to actual TSDF locations, and (2) a second time where no constraints were put on the metro/nonmetro distribution of the random points. All three replications yielded similar results. 


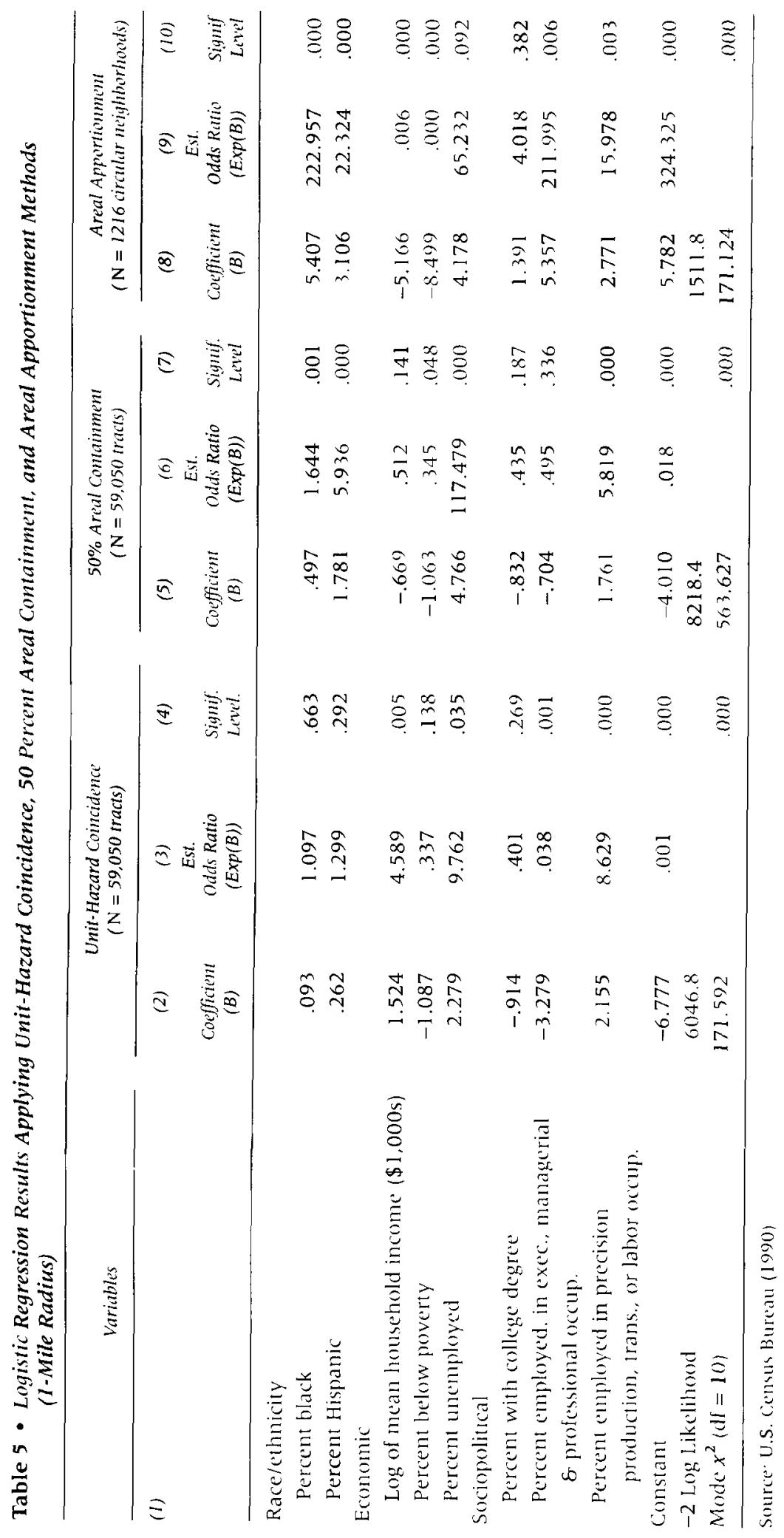


land use is believed to be non-linearly related to income (Been 1995; Pastor, Sadd, and Morello-Frosch 2004), diminishing sharply with rising income, we furthermore adopted Manuel Pastor, Jim Sadd, and Rachel Morello-Frosch's (2004) approach of entering the log of mean household income into the regression analyses. ${ }^{6}$

Examination of the results in Table 5 reveals important differences obtained from applying unit-hazard coincidence and distance-based methods. For example, when applying the unit-hazard coincidence method, the African American and Hispanic percentages of the census tracts are not at all significant predictors of the location of TSDFs (see column 4), while percent employed in management/professional occupations and percent employed in precision production/labor are the strongest predictors (with significance levels of .001 and .000 , respectively). Percent unemployed is also statistically significant (alpha $=.035$; other nonrace variables are either not significant or predict TSDF location in the unexpected direction). Such results may be interpreted to mean that any disparities found between minority and white communities in the distribution of the nation's TSDFs may largely be a function of the disparities in political clout between "blue" and "white collar" communities in keeping out such facilities, rather than that African American and Hispanic communities are necessarily targeted for TSDFs or that housing discrimination keeps some African Americans and Hispanics from moving out while steering others toward the TSDFs.

In contrast, when the 50 percent areal containment method is applied, the African American and Hispanic percentages of the tracts become highly statistically significant predictors of TSDF location (at .001 and .000 levels of significance; see column 7, Table 5). The statistical significance of the race variables increases at the same time that two of the economic variables, percent living in poverty and percent unemployed, also increase in statistical significance (alphas $=.048$ and .000 , respectively). Percent employed in precision production/labor occupations also remains highly statistically significant, although percent employed in management/professional occupations is no longer so. Similar results are obtained using the areal apportionment method, except that percent unemployed does not quite reach the .05 level of significance. Thus, in contrast to the earlier interpretations using the unit-hazard coincidence method, the results obtained by using the two distance-based methods may be taken to mean that, although there is evidence to indicate that economic and sociopolitical factors influence TSDF location, these factors by themselves do not entirely account for the racial disparities found in their distribution. Other factors related to race, such as racial targeting or housing discrimination, also appear to be playing a role.

\section{Summary and Conclusions}

Although it is the most widely used approach in conducting environmental inequality assessments, the unit-hazard coincidence method inadequately controls for proximity between environmentally hazardous sites and nearby populations. Rather than determining the precise geographic location of the site, this method only considers whether the site and a host unit are coincident and assumes people living in the host unit are closer to the site than people living in non-host units. Not taken into account, however, is the proximity of the site to nearby units. Even though people in nearby units may be as near to the site as those in the host tract proper, nearby units are considered to be no different demographically than nonhost units much farther away. Also not taken into account is the large variation in the sizes of host units. Even though people in large host tracts may be dispersed quite far from the hazardous sites in them, large host units are given the same weight as small host units in the analyses. If the proposition is true that hazardous sites are disproportionately located where

6. We also conducted the logistic regression analyses using the non-transformed mean household income and found no appreciable differences in the pattern of results. 
minorities and poor people live, then reliance on the unit-hazard coincidence method has likely led to underestimations of the actual magnitude of racial and socioeconomic disparities around a wide variety of environmentally hazardous sites. Furthermore, assessments about the relative importance of various factors thought to account for the disparities have also likely been affected.

Distance-based methods, such as the 50 percent areal containment and areal apportionment methods, represent a significant improvement over the unit-hazard coincidence method in controlling for proximity between environmentally hazardous sites and nearby residential populations. These methods take into account the precise geographic locations of hazardous sites and sort units or parts of units-whether host or non-host-that are within a specified distance of these sites from those that lie beyond the distance. Distance-based methods not only better control for proximity around environmentally hazardous sites than does the unit-hazard coincidence method, they also assure greater consistency in the size and shape of the geographic areas around the hazard, and greater consistency in the location of the hazards within these areas. We also note the considerable robustness in the results obtained by the 50 percent areal containment and areal apportionment methods. The results obtained are very similar regardless of which of these two distance-based methods is employed. Results are also very similar regardless of which predefined geographic unit is used as the building block unit: block groups, census tracts, or zip code areas.

We demonstrated that when racial disparities around the nation's TSDFs are analyzed applying distance-based methods, such disparities are found to be much greater than when the unit-hazard coincidence method is applied. Although minorities made up only a quarter of the nation's population in 1990, over 40 percent of the population living within 1 mile of hazardous waste TSDFs were persons of color. The nearly 20 percent difference $(43.2$ percent compared to 24 percent; see columns 2 and 5 in Table 3 ) in the minority percentages between host and non-host neighborhoods within 1.0 mile of a TSDF is clearly much greater than the 1 percent to 3 percent differences that are found when the unit-hazard coincidence method is applied. Even at a distance of 3 miles, the difference in the proportion of nonwhites in host and non-host neighborhoods is found to be greater.

Furthermore, distance-based methods lead to different assessments about the relative importance of economic and sociopolitical factors in accounting for racial disparities in the distribution of the nation's TSDFs. When logistic regression analysis is performed with unithazard coincidence, the African American and Hispanic percentages of tracts appear to have no independent effect on the location of TSDFs beyond what can be explained by differences in economic and sociopolitical variables. When 50 percent areal containment and areal apportionment are applied, racial disparities in the distribution of the nation's TSDFs persist despite controlling for the economic and sociopolitical make-up of the tracts, suggesting that factors uniquely associated with race, such as racial targeting at the time of siting, housing segregation after siting, and institutional forms of discrimination may play a role in presentday TSDF locations. Determining precisely what factors play a role in present-day racial disparities in industrial and hazardous waste facility location will of course require further study. Nevertheless, our analysis demonstrates that future quantitative studies employing multivariate analyses to test hypotheses about cause and effect relationships will likely yield different results from many prior studies where proximity between hazardous sites and nearby populations were not adequately controlled.

Finally, even though measuring proximity to environmentally hazardous sites is not the same as measuring actual exposure to health risks, the methods we describe are relevant for examining racial and socioeconomic disparities in the dispersion of pollution risks as well. Pollution dispersion and risk assessment techniques involve measuring the types and quantities of toxic emissions, timing of release, meteorological conditions affecting emission dispersion (such as wind speed and direction), and other factors so that fallout patterns ("pollution footprints") and their level of risk can be determined (Andrews 2003, Ash and Fetter 2004, Chakraborty 
and Armstrong 1997; Glickman et al. 1995, Hamilton 1999). Fifty percent areal containment and areal apportionment methods can be applied to determine which geographic units or portions of units fall within the footprint boundaries. The demographic characteristics of the aggregated units within the footprint boundaries can then be compared with those outside the boundaries (see, e.g., Glickman et al. 1995; and Chakraborty and Armstrong 1997).

Currently, relatively few environmental justice studies have involved pollution dispersion and risk assessment modeling, largely because of the difficulties in acquiring the necessary data for them, particularly when a great number of polluting facilities are examined as in national studies such as ours. Because of the uncertainties of such modeling associated with the incompleteness of data about the type, quantity, and timing of toxic releases, meteorological conditions, fallout patterns, etc. (Andrews 2003), the advantages of more straightforward proximity measures ought not to be overlooked. They provide "hard numbers" about the location of environmentally hazardous sites and their proximity to nearby residential populations. And although at best they are only an indirect measure of potential health risk, they nevertheless indicate the presence of other probable quality of life impacts of concern to nearby residents, including visual blight, noise, noxious odors, traffic congestion, depressed property values, social stigmatization, and others (Edelstein 2004; Mohai 1995; Mohai and Bryant 1998).

The prominence of debates in both academic and political arenas about the role of race in the distribution of environmental costs and benefits and the importance of studies attempting to gain a better understanding of the role race plays in society in general underscore the need for applying the best methodologies available. Whether pollution dispersion assessments or site proximity assessments are seen as the best ways to evaluate environmental injustices, the goal in either case will be to best match the location of the potentially affected or exposed populations with that of the environmental hazard. We believe that distance-based methods provide distinct improvements in making such matches over previous methods, such as the unit-hazard coincidence method. Furthermore, given the current widespread availability of GIS technology, the application of distance-based methods should be feasible even for national level environmental inequality studies.

\section{Appendix}

Tables A.? and A.2 provide the results of statistical analyses of the differences in demographic values between host and non-host neighborhoods defined by 50 percent areal containment and areal apportionment methods. In conducting statistical tests involving the 50 percent areal containment method, the analyses were performed three times, once each time using block groups, census tracts, and zip code areas, respectively, as the geographic units of analysis. Because neighborhoods beyond the 1- and 3-mile distances from TSDFs make up the vast majority of the geographic areas (over 99 percent of either block groups, tracts, or zip code areas), the demographic values of the combined populations within these units were treated as constants in one-sample $t$-tests (see Table A.1). It was found that all differences between host and non-host neighborhood (defined by either 1 or 3 buffers) were statistically significant at the .05 level, except for mean property values when zip code areas are used as the units of analysis.

In conducting statistical tests involving the areal apportionment method, the units of analysis were either 1 or 3 mile perfectly circular neighborhoods around the nation's TSDFs. These perfectly circular neighborhoods were constructed three times, once using block groups as the building block units, again using tracts as the building blocks, and a third time using zip code areas as the building blocks. As with the 50 percent areal containment method, demographic values for areas beyond the 1 - and 3-mile circular neighborhoods were treated as constants in one-sample t-tests (see Table A.2). As before, this was done because the vast majority of the area and population in the United States lies beyond the 1 - and 3-mile host areas. Moreover, it would have been difficult to represent non-host areas using 1- or 3-mile circular buffers, other 

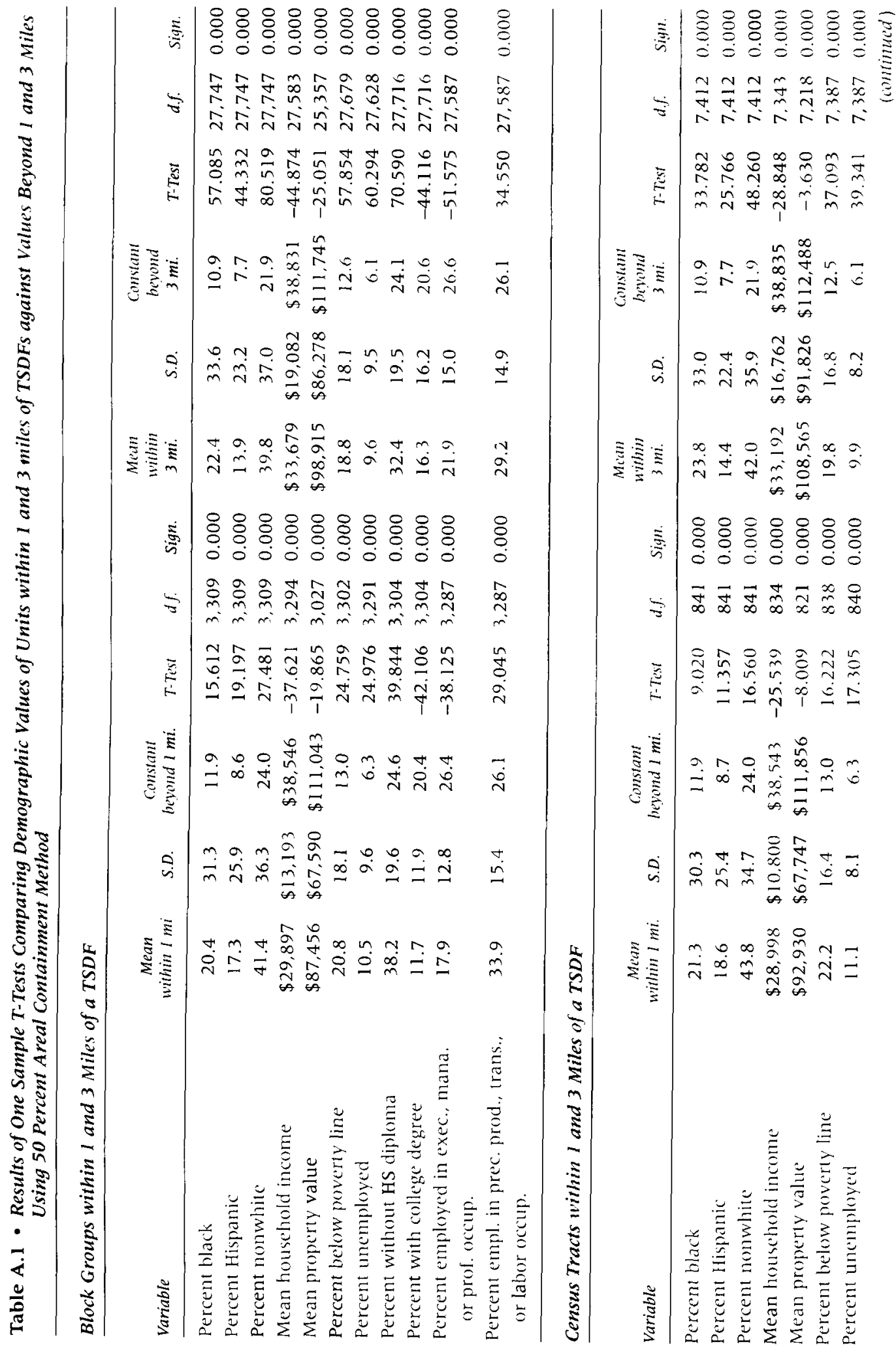


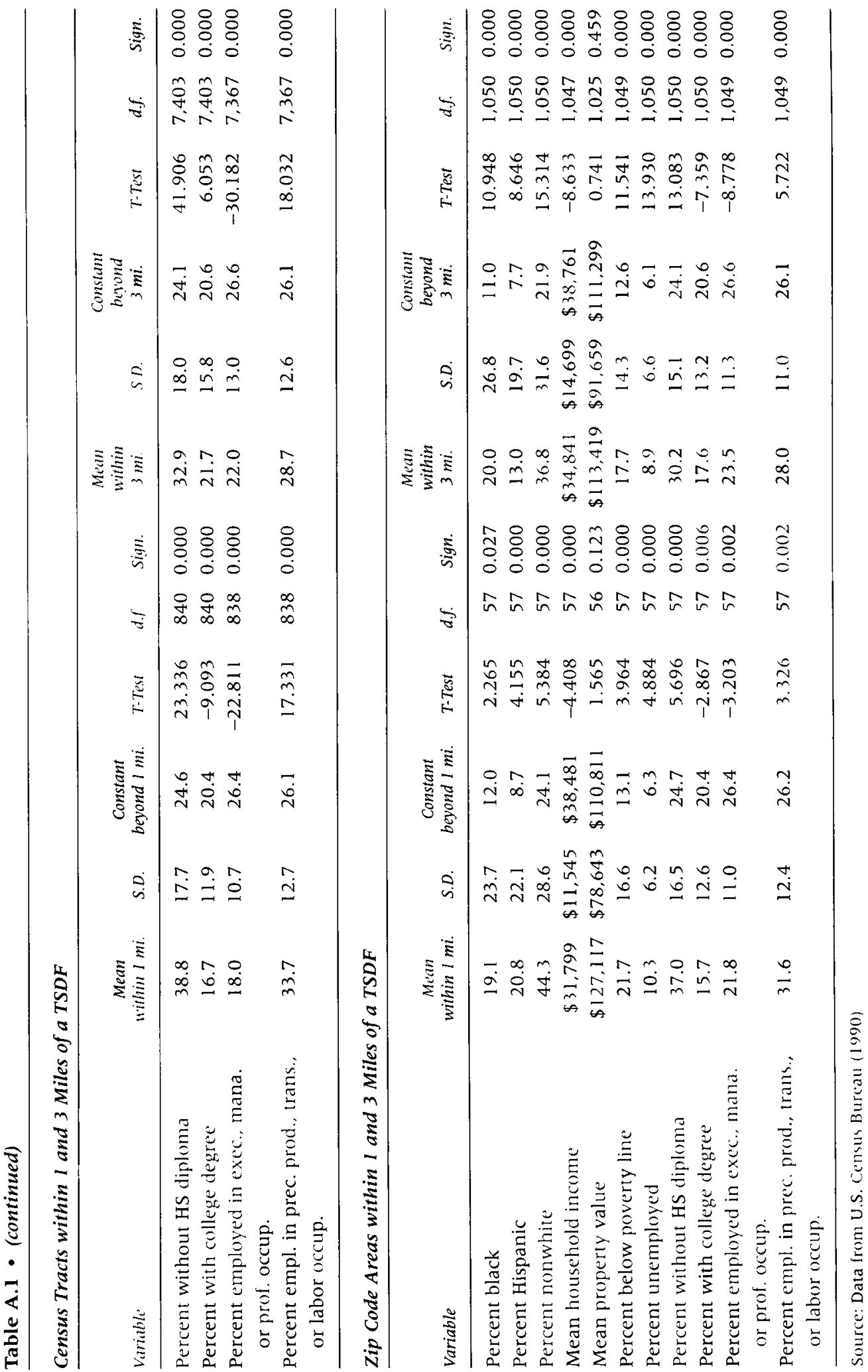



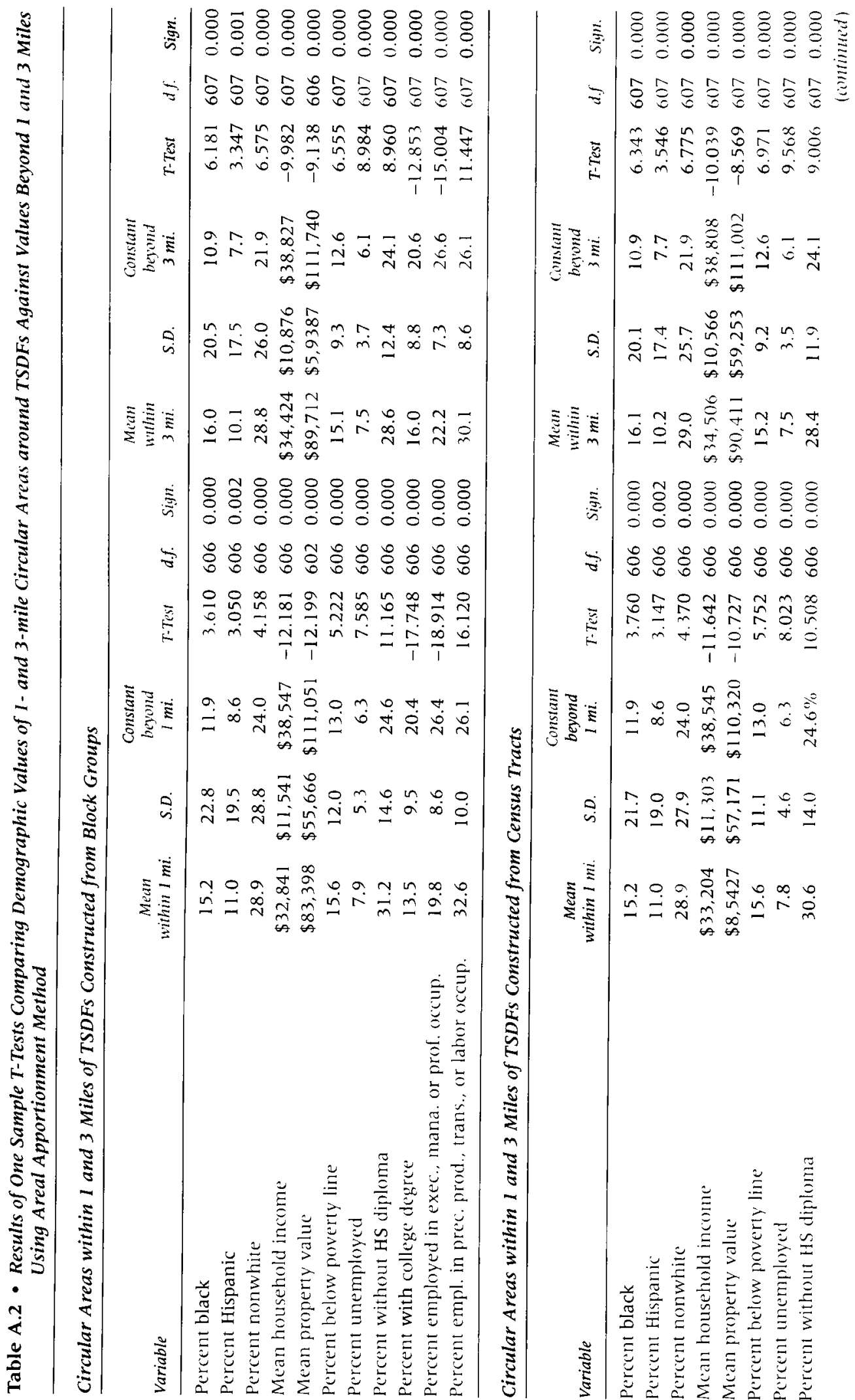


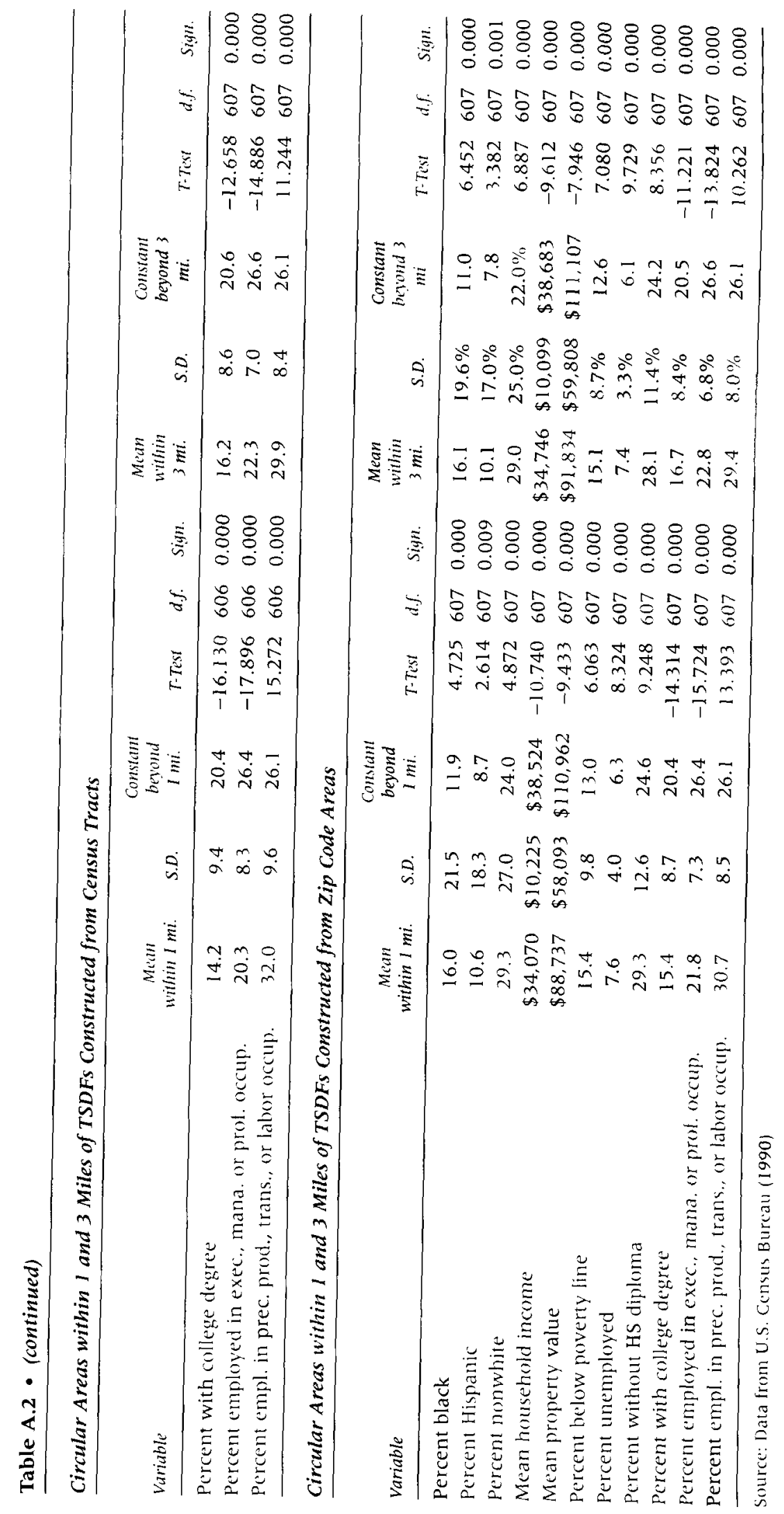


than by locating a random sample of points outside the host areas and constructing buffers around them (as we did for the logistic regression in Table 5), However, we wanted to include demographic values for the entire United States rather than for only a sample of areas. It was found that all differences in demographic values between host and non-host neighborhood (defined by either 1 or 3 buffers) were statistically significant at the .05 level.

\section{References}

Allen, Barbara L. 2003. Uneasy Alchemy: Citizens and Experts in Louisiana's Chemical Corridor Disputes. Cambridge, MA: The MIT Press.

Anderton, Douglas L., Andy B. Anderson, John Michael Oakes, and Michael R. Fraser. 1994. "Environmental Equity: The Demographics of Dumping." Demography 31:229-48.

Andrews, Richard N. L. 2003. "Risk-Based Decision Making." Pp. 223-48 in Environmental Policy: New Directions for the 21st Century, Sth Ed, edited by N. J. Vig and M. E. Kraft. Washington, DC: CQ Press.

Ash, Michael and T. Robert Fetter. 2004. "Who Lives on the Wrong Side of the Environmental Tracks? Evidence from the EPA's Risk-Screening Environmental Indicators Model." Social Science Quarterly 85:441-62.

Been, Vicki. 1995. "Analyzing Evidence of Environmental Justice." Journal of Land Use $\theta$ Law 1 1:1-36.

Been, Vicki and Frances Gupta. 1997. "Coming to the Nuisance or Going to the Barrios? A Longitudinal Analysis of Environmental Justice Claims." Ecology Law Quarterly 24:1-56.

Beggs, John J. 1995. "The Institutional Environment: Implications for Race and Gender Inequality in the U.S. Labor Market." American Sociological Review 60:612-33.

Boer, J. Tom, Manuel Pastor, James L. Sadd, and Lori D. Snyder. 1997. "Is There Environmental Racism? The Demographics of Hazardous Waste in Los Angeles." Social Science Quarterly 78:793-810.

Boone, Christopher G. and Ali Modarres. 1999. "Creating a Toxic Neighborhood in Los Angeles County: A Historical Examination of Environmental Inequality." Urban Affairs Review 35:163-87.

Brook, Daniel. 1998. "Environmental Genocide: Native Americans and Toxic Waste." The American Journal of Economics and Sociology 57:105-13.

Brown, Phil. 1997. "Popular Epidemiology Revisited." Current Sociology 45:137-56.

Bryant, Bunyan and Paul Mohai. 1992. Race and the Incidence of Environmental Hazards: A Time for Discourse. Boulder, CO: Westview Press.

Bullard, Robert D. 1990. Dumping in Dixie: Race, Class, and Environmental Quality. Boulder, CO: Westview Press.

Bullard, Robert D. and Beverly Hendrix Wright. 1987. "Environmentalism and the Politics of Equity: Emergent Trends in the Black Community." Mid-American Review of Sociology 12(2):21-37.

Cable, Sherry and Michael Benson. 1993. "Acting Locally: Environmental Injustice and the Emergence of Grass-Roots Environmental Organizations." Social Problems 40:464-77.

Camacho, David E. 1998. Environmental Justice, Political Struggles: Race, Class, and the Environment. Durham, NC: Duke University Press.

Cancio, A. Silvia, T. David Evans, and David J. Maume, Jr. 1996. "Reconsidering the Declining Significance of Race: Racial Differences in Early Career Wages." American Sociological Review 61:541-56.

Chakraborty, Jayajit and Marc P. Armstrong. 1997. "Exploring the Use of Buffer Analysis for the Identification of Impacted Areas in Environmental Equity Assessment." Cartography and Geographic Information Systems 24: 1 45-57.

Clark, Brett. 2002. "The Indigenous Environmental Movement in the United States." Organization $\theta$ Environment 15:410-42.

Cole Luke W. and Sheila R. Foster. 2001. From the Ground Up: Environmental Racism and the Rise of the Environmental Justice Movement. New York and London: New York University Press.

Commission for Racial Justice (CRJ). 1987. Toxic Waste and Race in the United States: A National Report on the Racial and Socioeconomic Characteristics of Communities with Hazardous Waste Sites. New York: United Church of Christ.

Daniels, Glynis and Samantha Friedman. 1999. "Spatial Inequality and the Distribution of Industrial Toxic Releases: Evidence from the 1990 TRI." Social Science Quarterly 80:244-62. 
Davidson, Pamela and Douglas L. Anderton. 2000. “The Demographics of Dumping II: Survey of the Distribution of Hazardous Materials Handlers." Demography 37:461-66.

Dolk, H., M. Vrijeid, B. Armstrong, L. Abramsky, F. Bianchi, E. Garne, V. Nelen, E. Robert, J. E. S. Scott, D. Stone, and R. Tenconi. 1998. "Risk of Congenital Anomalies Near Hazardous-Waste Landfill Sites in Europe: The EUROHAZCON Study." The Lancet 352:423-27.

Edelstein, Michael R. 2004. Contaminated Communities: Coping with Residential Toxic Exposure. $2 \mathrm{~d}$ ed. Cambridge MA: Westview Press.

Eggebeen, David J. and Daniel T. Lichter. 1991. "Race, Family Structure, and Changing Poverty among American Children." American Sociological Review 56:801-17.

Foreman, Christopher. 1998. The Promise and Peril of Environmental Justice. Washington, DC: Brookings Institution Press.

Freudenburg, William R. 1997. "Contamination, Corrosion and the Social Order: An Overview." Current Sociology 45(3):19-39.

Geolytics, Inc. 1998. CensusCD+Maps Version 3.0. New Brunswick, NJ: GeoLytics Inc. . 1999. StreetCD 98. New Brunswick, NJ: Geolytics Inc..

Geschwind, Sandra A., Jan Stolwijk, Michael Bracken, Edward Fitzgerald, Alice Stark, Carolyn Olsen, and James Melius. 1992. "Risk of Congenital Malformations Associated With Proximity to Hazardous Waste Sites." American Journal of Epidemiology 135:1197-207.

Glickman, Theodore. 1994. "Measuring Environmental Equity with GIS." Renewable Resources Journal 12(3):17-21.

Glickman, Theodore S., Dominic Golding, and Robert Hersh. 1995. "GIS-Based Environmental Equity Analysis: A Case Study of TRI Facilities in the Pittsburgh Area." Pp. 95-114 in Computer Supported Risk Management, edited by G. E. G. Beroggi and W. A. Wallace. Dordrecht, Netherlands: Kluwer Academic.

Goldman, Benjamin A. and Laura Fitton. 1994. Toxic Wastes and Race Revisited: An Update of the 1987 Report on the Racial and Socioeconomic Characteristics of Communities with Hazardous Waste Sites. Washington, DC: Center for Policy Alternatives.

Hamilton, James T. 1995. "Testing for Environmental Racism: Prejudice, Profits, Political Power?" Journal of Policy Analysis and Management 14:107-32.

- 1999. "Exercising Property Rights to Pollute: Do Cancer Risks and Politics Affect Plant Emission Reductions?" Journal of Risk and Uncertainty 18:105-24.

Hamilton, James T. and W. Kip Viscusi. 1999. Calculating Risks? The Spatial and Political Dimensions of Hazardous Waste Policy. Cambridge, MA: MIT Press.

Hayward, Mark D., Eileen M. Crimmins, Toni P. Miles, and Yu Yang. 2000. "The Significance of Socioeconomic Status in Explaining the Racial Gap in Chronic Health Conditions." American Sociological Review 65:910-30.

Hird, John A. and Michael Reese. 1998. "The Distribution of Environmental Quality: An Empirical Analysis." Social Science Quarterly 79:694-716.

Hughes, Michael and Melvin E. Thomas. 1998. "The Continuing Significance of Race Revisited: A Study of Race, Class, and Quality of Life in America, 1972 to 1996." American Sociological Review 63:785-95.

Hurley, Andrew. 1995. Environmental Inequities: Class, Race and Industrial Pollution in Gary, Indiana, 19451990. Chapel Hill: University of North Carolina Press.

- 1997. "Fiasco at Wagner Electric: Environmental Justice and Urban Geography in St. Louis." Environmental History 2:460-81.

James, David R. and Holly J. McCammon. 1997. "Citizenship and Public Schools: Accounting for Racial Inequality in Education in the Pre- and Post-Disfranchisement South." American Sociological Review 62:34-52.

Jargowsky, Paul A. 1996. "Take the Money and Run: Economic Segregation in U.S. Metropolitan Areas." American Sociological Review 61:984-98.

Kohlhase, Janet E. 1991. "The Impact of Toxic Waste Sites on Housing Values." Journal of Urban Economics 30:1-26.

Lerner, Steve. 2005. A Struggle for Environmental Justice in Louisiana's Chemical Corridor. Cambridge, MA: The MIT Press.

Lester, James P., David W. Allen, and Kelly M. Hill. 2001. Environmental injustice in the United States: Myths and Realities. Boulder, $\mathrm{CO}$ : Westview Press.

McCall, Leslie. 2001. "Sources of Racial Wage Inequality in Metropolitan Labor Markets: Racial, Ethnic, and Gender Differences." American Sociological Review 66:520-41. 
Mohai, Paul. 1985. "Public Concern and Elite Involvement in Environmental-Conservation Issues." Social Science Quarterly 66:820-38.

- 1995. "The Demographics of Dumping Revisited: Examining the Impact of Alternate Methodologies in Environmental Justice Research." Virginia Environmental Law Journal 14:61 5-52.

Mohai, Paul and Bunyan Bryant. 1992. "Environmental Racism: Reviewing the Evidence." Pp. 163-76 in Race and the Incidence of Environmental Hazards: A Time for Discourse, edited by B. Bryant and P. Mohai. Boulder, CO: Westview Press.

. 1998. “Is There a 'Race' Effect on Concern for Environmental Quality?” Public Opinion Quarterly 62:475-505.

Mohai, Paul and Robin Saha. 1994. "Environmental Injustice: Examining Altemative Explanations." Paper presented at the 1994 Meeting of the American Sociological Association, August, Los Angeles, California.

- 2006. "Reassessing Racial and Socioeconomic Disparities in Environmental Justice Research." Demography 43:383-99.

Montrie, Chad. 2005. "From Dairy Farms to Housing Tracts: Environment and Race in the Making of a Memphis Suburb." Journal of Urban History 31:219-40.

Nelson, Arthur C., Michelle Genereux, and John Genereux. 1992. "Price Effects and Landfills on House Values." Land Economics 68:359-65.

Oakes, John Michael, Douglas L. Anderton, and Andy B. Anderson. 1996. "A Longitudinal Analysis of Environmental Equity in Communities with Hazardous Waste Facilities." Social Science Research 25:125-48.

Pastor, Manuel, Jim Sadd, and John Hipp. 2001. "Which Came First? Toxic Facilities, Minority Move-In, and Environmental Justice." Journal of Urban Affairs 23:1-21.

Pastor, Manuel, Jim Sadd, and Rachel Morello-Frosch. 2004. "Waiting to Inhale: The Demographics of Toxic Air Releases in 2 I st Century California." Social Science Quarterly 85:420-40.

Pellow, David N. 2001. "Environmental Justice and the Political Process: Movements, Corporations, and the State." The Sociological Quarterly 42:47-67.

- 2002. Garbage Wars: The Struggle for Environmental Justice in Chicago Cambridge, MA: MIT Press.

Pollock, Philip H. and M. Elliot Vittas. 1995. "Who Bears the Burdens of Environmental Pollution? Race. Ethnicity, and Environmental Equity in Florida." Social Science Quarterly 76:294-310.

Pulido, Laura. 1996a. "A Critical Review of the Methodology of Environmental Racism Research." Antipode 28:142-59.

1996b. Environmentalism and Economic Justice: Two Chicano Struggles in the Southwest. Tucson, AZ: University of Arizona Press.

Pulido, Laura, Steve Sidawi, and Robert O. Vos. 1996. "An Archaeology of Environmental Racism in Los Angeles." Urban Geography 17:419-39.

Rechtschaffen, Clifford and Eileen Gauna, eds. 2002. Environmental Justice: Law, Policy, and Regulation. Durham, NC: Caroline Academy Press.

Rhodes, Edwardo Lao, 2003. Environmental Justice in America: A New Paradigm. Bloomington and Indianapolis: Indiana University Press.

Ringquist, Evan J. 2003. "Environmental Justice: Normative Concerns and Empirical Evidence." Pp. 249-73 in Environmental Policy: New Directions for the 21 st Century. 5 th ed., edited by N. J. Vig and M. E. Kraft. Washington, DC: CQ Press.

- 2005. "Assessing Evidence of Environmental Inequities: A Meta-Analysis." Journal of Policy Analysis and Management 24:223-47.

Saha, Robin and Paul Mohai. 2005. "Historical Context and Hazardous Waste Facility Siting: Understanding Temporal Patterns in Michigan." Social Problems 52:618-48.

Schlosberg, David. 1999. Environmental Justice and the New Pluralism: The Challenge of Difference for Environmentalism. New York: Oxford University Press.

Sheppard, Eric, Helga Leitner, Robert B. McMaster, and H. Tian. 1999. "GIS-Based Measures of Environmental Equity: Exploring Their Sensitivity and Significance." Journal of Exposure Analysis and Environmental Epidemiology 9:1 8-28.

Smith, David Horton and Jacqueline Macaulay. 1980. Participation in Social and Political Activities. San Francisco: Jossey-Bass.

Stretesky Paul and Michael J. Hogan. 1998. "Environmental Justice: An Analysis of Superfund Sites in Florida." Social Problems 45:268-87.

Szasz, Andrew. 1995. EcoPopulism: Toxic Waste and the Movement for Environmental Justice. Minneapolis: University of Minnesota Press. 
Szasz, Andrew and Michael Meuser. 2000. "Unintended, Inexorable: The Production of Environmental Inequalities in Santa Clara County, California." American Behavioral Scientist 43:602-32.

Taylor, Dorceta E. 2000. "The Rise of the Environmental Justice Paradigm." American Behavioral Scientist 43:508-80.

U.S. Census Bureau. 1990. 1990 Census of Housing, Summary Tape File 1 Technical Documentation: Appendix AArea Classifications. Washington, DC: U.S. Government Printing Office.

U.S. General Accounting Office (GAO). 1983. Siting of Hazardous Waste Landfills and Their Correlation With Racial and Economic Status of Surrounding Communities. RCED-83-168. Washington DC: U.S. General Accounting Office.

Wessex Inc. [1992] 1994. 1990 Census of Population and Housing. Summary Tape Files 1A and 3A [CDROMs]. Washington, DC: U.S. Bureau of the Census [producer] 1992. Winnetka, IL: Wessex, Inc. [distributor] 1994.

Wilson, William. 1987. The Truly Disadvantaged. Chicago: University of Chicago Press. 